\title{
Towards a Theoretical Background for Strong-Scattering Inversion - Direct Envelope Inversion and Gel'fand-Levitan-Marchenko Theory
}

\author{
Ru-Shan $\mathrm{Wu}^{1, *}$ \\ ${ }^{1}$ Modeling and Imaging Laboratory, Earth and Planetary Sciences, University of \\ California, Santa Cruz, 95060, CA, USA.
}

Received 21 April 2018; Accepted (in revised version) 8 November 2018

\begin{abstract}
Strong-scattering inversion or the inverse problem for strong scattering has different physical-mathematical foundations from the weak-scattering case. Seismic inversion based on wave equation for strong scattering cannot be directly solved by Newton's local optimization method which is based on weak-nonlinear assumption. Here I try to illustrate the connection between the Schrödinger inverse scattering (inverse problem for Schrödinger equation) by GLM (Gel'fand-Levitan-Marchenko) theory and the direct envelope inversion (DEI) using reflection data. The difference between wave equation and Schrödinger equation is that the latter has a potential independent of frequency while the former has a frequency-square dependency in the potential. I also point out that the traditional GLM equation for potential inversion can only recover the high-wavenumber components of impedance profile. I propose to use the Schrödinger impedance equation for direct impedance inversion and introduce a singular impedance function which also corresponds to a singular potential for the reconstruction of impedance profile, including discontinuities and long-wavelength velocity structure. I will review the GLM theory and its application to impedance inversion including some numerical examples. Then I analyze the recently developed multiscale direct envelope inversion (MS-DEI) and its connection to the inverse Schrödinger scattering. It is conceivable that the combination of strong-scattering inversion (inverse Schrödinger scattering) and weak-scattering inversion (local optimization based inversion) may create some inversion methods working for a whole range of inversion problems in geophysical exploration.
\end{abstract}

AMS subject classifications: 74J20, 86A15

Key words: Strong-scattering, strong nonlinear inversion, GLM theory, envelope inversion.

*Corresponding author. Email address: rwu@ucsc.edu (R. S. Wu) 


\section{Introduction}

The earth medium has a very nonlinear response to the excitation of seismic waves. Therefore, the parameter inversion, especially velocity inversion, is expected generally to be nonlinear for seismic exploration. Newton's scheme of local optimization has the assumption of weak nonlinearity so that the nonlinear search can be realized by a local linearization [1]. It is well-known that the local minimization approach has many difficulties, such as the cycle-skipping, local minima, or divergence of the iterative process (for a review, see [2]). On the other hand, we know that Born data has a simple linear relationship with the parameter change no matter how strong the perturbations are. Born scattering observes linear superposition, so the final scattered field is the superposition of all the scattered fields by each element volume of the whole medium. If the data collected are the Born data, everything will be simple. For a full illumination, the inversion problem can be solved by a linear inversion. However, the real scattering process in the earth is nonlinear, and the strong interaction and interference between scattered waves including multiply scattered waves from different elements destroy the linearity of the Born data. For strong-nonlinear data due to strong scattering, the linearity of the data response cannot be recovered by any linear process. This why the Newton type inversion has to start the iterative process in a nearly linear region. That means the iterative process must start with a "good" initial model which is "close" to the true model.

Different from the popular Newton's method, there are some special approaches which can solve strong-scattering inversion without weak-scattering assumption. One approach is the direct nonlinear inversion or nonlinear inverse scattering based on wave equation. Since the Newton's local optimization method uses the linearized sensitivity operator, one approach to deal with strong nonlinearity is to introduce directly the nonlinear sensitivity operator (NLSO) to the inversion. Wu and Zheng [3] proved that the higher-order terms of the NLSO, i.e. the higher order Fréchet derivatives correspond

to the higher-order multiple-scattering terms in the scattering series. Scattering series in solving forward and inverse scattering problem has been known for a long time in the literature. However, in the case of strong-scattering for wave equation, the conventional Born-Neumann series may not converge (see e.g., [4]), which is the cause for the divergence or falling into local minima of nonlinear inversion. To overcome the divergence problem of scattering or inverse-scattering series, renormalization or renormalization group (RG) theory/method was applied to the scattering or inverse-scattering series in different ways. One way is to use the Volterra integral to renormalize the scattering series [5,6]. Yao et al. [6] uses a perturbation series approach and calculated the Volterra inverse scattering series (VISS) to the third order, but the convergence of the VISS is not discussed. Weglein et al. [7] proposed to use different task-oriented subseries to mitigate the divergence problem. The other way to renormalize the scattering series is to use the De Wolf series [3, 8-11]. Geng et al. [12] has applied the method to inversion using up to the second order NLSO.

Another approach for nonlinear inversion, especially for strong-nonlinear inversion 
(strong inverse-scattering) is to formulate the strong-scattering by using the Schrödinger equation. This approach borrows theory/methods from quantum scattering. Inversescattering based on the Schrödinger equation is among the most popular one. In Schrödinger equation, the scattering potential is frequency-independent, that is an important difference from the wave equation where the scattering potential has a frequencysquare dependence. This is a critical factor for the applicability of some theory / method. Schrödinger equation is devised by Schrödinger in the early stage of quantum mechanics to formulate the wave-particle duality: propagate like wave but interaction like particle. The equation can be applied to field interaction with nucleus, atom or molecular. So the scattering involved is "strong" interaction, in which the potential is like a fixed barrier whose property does not change during the interaction. For classic wave scattering, such as acoustic, elastic or electro-magnetic waves, normally the scattering is frequency-dependent, so the interaction will change the property of the potential. For strong-contrast and large-body scattering, such as boundary scattering, it can be treated as strong-scattering or "Schrödinger scattering". We will discuss it in more details in the second section. For the one-dimensional Schrödinger equation, there is an exact solution for the inverse problem from the celebrated and elegant GLM (Gel'fand-LevitanMarchenko) theory. Nevertheless, although there is a corresponding theory for the 3D potential scattering [60,61], multi-dimensional inverse scattering for impedance and velocity scattering in wave equation is not available yet. So the GLM theory has very limited application to 3D wave inverse-scattering problem.

The third approach is to nonlinearly extract the slow-dynamics of the waveform data so that the gross property (large-scale structure, or the low-wavenumber component) can be inverted from the derived data: envelope data. Then the waveform data are used for the fine-scale structure (high-wavenumber components). This is the recently developed waveform envelope inversion or seismic envelope inversion (SEI) [13-17]. Envelope inversion can partly solve the starting-model dependence problem of FWI (full waveform inversion). However, for case of strong-nonlinear FWI caused by strong-scattering, such as the waveform inversion involved large salt-structures, the conventional SEI is still helpless. The newly-introduced direct envelope inversion (DEI) [18-24] opens a new door for strong-nonlinear waveform inversion. Envelope data has rich ULF (ultra-low frequency) information which is critical for the gross structure recovery. Direct envelope inversion (DEI) is based on the strong-scattering physics and envelope data can be considered as transformed (by demodulation operator) from waveform data to energy reflection data [24]. In this paper, I try to establish the link and similarity between the Schrödinger inverse-scattering and the direct envelope inversion. As we can see that both GLM inversion and envelope inversion belong to a category of strong-scattering inversion, and the problem can be related to the inverse problem for Schrödinger equation (inverse Schrödinger scattering). For this purpose, I'll first review the GLM theory and the recent development when applied to seismic impedance inversion. The connection and comparison between GLM theory and direct envelope inversion is preliminary, but hope to shed some light for future development of 3D strong-scattering inversion in both theory and applications. 
The paper is organized as follows. Section 1 is the introduction. In Section 2, I will discuss the GLM theory for 1-D Schrödinger equation and its application to acoustic impedance inversion. In order to apply the GLM theory to impedance inversion including discontinuities, a singular function is introduced to deal with singularities. Some numerical examples are given to demonstrate the recovery of impedance profile especially the long-wavelength components. Liouville transform and it inverse are discussed as a renormalization procedure. In Section 3, the three-dimensional impedance inversion is discussed and a 3-D Schrödinger-like equation is introduced through the Kirchhoff approximation (physical optics approximation). The success of the method is demonstrated by numerical examples for the SEG/EAGE salt model. Then the link between the direct envelope inversion and GLM theory for inverse Schrödinger scattering is discussed, especially the similarity of envelop data with the time-series of scattering amplitude (reflection coefficient) in the 1-D case. Finally, discussion and conclusion are given in Section 4 .

\section{Exact 1-D inversion by GLM theory}

\subsection{Acoustic wave equation and impedance Schrödinger equation}

For the acoustic wave problem, the 1-D wave equation in the time domain is

$$
\frac{\partial}{\partial z}\left(\frac{1}{\rho(z)} \frac{\partial}{\partial z} p(z, t)\right)-\frac{1}{\kappa(z)} \frac{\partial^{2}}{\partial t^{2}} p(z, t)=0,
$$

where $z$ is the spatial coordinate (depth), $p$ is the pressure field, $\rho$ and $\kappa$ are the density and bulk modulus of the medium, respectively.

Following Ware and Aki [25], we can change the above wave equation into a Schrödinger equation with certain potential representing the parameter variations. First we change the space variable $z$ into a traveltime-correspondent location parameter $\varsigma$ according to

$$
d \varsigma=d z / c(z)
$$

where $c(z)$ is wave propagation speed defined as

$$
c=\sqrt{\kappa / \rho} .
$$

This $z-\varsigma$ transform is the Liouville transform which incorporates the velocity variation along the path into the $\varsigma$ variable (coordinate stretching-shrinking according to local velocity). The other variable transform is to change the pressure field $p(z, t)$ into an energyflux normalized field which observes the energy conservation law when interacting with boundaries,

$$
\psi(z, t)=p(z, t) / \sqrt{\rho(z) c(z)}=p(z, t) \eta(z),
$$


where $\rho c$ is the impedance and

$$
\eta(z)=1 / \sqrt{\rho(z) c(z)} .
$$

With the variable transforms of (2.2) and (2.4), the acoustic equation (2.1) can be transformed into

$$
\left(\frac{\partial^{2}}{\partial \varsigma^{2}}-\frac{\partial^{2}}{\partial t^{2}}\right) \psi(\varsigma, t)-\left(\frac{1}{\eta(\varsigma)} \frac{\partial^{2}}{\partial \varsigma^{2}} \eta(\varsigma)\right) \psi(\varsigma, t)=0 .
$$

This is a Schrödinger impedance equation (Chadan and Sabatier, [26]). The above equation can be also written as a typical Schrödinger equation for scattering potential

$$
\begin{aligned}
& \left(\frac{\partial^{2}}{\partial \varsigma^{2}}-\frac{\partial^{2}}{\partial t^{2}}\right) \psi(\zeta, t)=q(\varsigma) \psi(\zeta, t)+s(\zeta, t), \\
& q(\varsigma)=\left(\frac{1}{\eta(\zeta)} \frac{\partial^{2}}{\partial \zeta^{2}} \eta(\zeta)\right),
\end{aligned}
$$

where $q(\varsigma)$ is the scattering potential, $q(\varsigma) \psi(\zeta, t)$ is the scattering secondary source and $s(s, t)$ is the real source (primary source).

Before discussing the GLM theory, let us first discuss the important differences between the Schrödinger equation and the wave equation. In the frequency domain, the Schrödinger equation (2.7) becomes

$$
\left(\frac{\partial^{2}}{\partial \varsigma^{2}}+\omega^{2}\right) \psi(\varsigma, \omega)=q(\varsigma) \psi(\varsigma, \omega)
$$

The RHS term is the equivalent source generated by scattering potential $q(\varsigma)$. We note that the potential $q$ is independent of frequency. So for $\mathrm{h}-\mathrm{f}^{\prime} \mathrm{s}$ the scattering term $q(\varsigma) \psi(\varsigma, \omega)$ will be much smaller than the background term $\omega^{2} \psi(\varsigma, \omega)$, and therefore the Born term (single-reflection approximation) will be dominant. In the limiting case, the Born approximation at $\mathrm{h}-\mathrm{f}$ will be accurate enough for the solution, and no l-f information is needed. This is very different from the wave question,

$$
\left(\frac{\partial^{2}}{\partial \zeta^{2}}+\omega^{2}\right) \psi(\varsigma, \omega)=\omega^{2} \varepsilon(\varsigma) \psi(\varsigma, \omega)
$$

where $\varepsilon(\varsigma)=n^{2}(\varsigma)-1=c_{0}^{2} / c^{2}(\varsigma)-1$ is the medium perturbation function (in this case, we set $c_{0}=1$ and take $\varsigma$ as the space variable). In the case of wave equation, the potential $\omega^{2} \varepsilon(\varsigma)$ is frequency-dependent and increases rapidly with frequency. In this case, to the contrary of Schrödinger equation, Born approximation of wave equation is valid only for low-frequencies.

The peculiar behavior of the Schrödinger equation (2.7) derived from the wave equation makes the inversion easier. We see that for the wave equation, the equivalent source is f-dependent, so the source inversion problem becomes a tough problem; while for the 
Schrödinger equation, the source inversion problem becomes overdetermined, a relatedly easy one. The key in converting the wave equation to Schrödinger equation is the Liouville transform, which changes the forward-scattering into a phase accumulation by renormalization. This will be discussed later in Section 2.6. Unfortunately, Liouville transform is not available in the 3-D case.

\subsection{GLM theory for potential and impedance inversions}

The GLM (Gel'fand-Levitan-Marchenko) theory [26-31] offers an exact solution for inverse problem of the 1-D Schrödinger equation in a form of integral equation (see also $[32,33])$

$$
K(\varsigma, t)=-R(t+\varsigma)-\int_{-\varsigma}^{\varsigma} K(\varsigma, \tau) R(t+\tau) d \tau, \quad \varsigma \geq 0, \quad-\varsigma \leq t \leq \varsigma,
$$

where $R(t)$ is the reflection record (reflection time series) on the surface, $K(\zeta, t)$ is an integral transform kernel (K-matrix in discrete form). Here we consider only the halfspace $(z \geq 0)$ problem with reflection time-series measured on the surface (assuming an absorbing boundary condition on the surface $z=0$ so that no reflection at the surface). The scattering potential can be recovered by

$$
q(\varsigma)=2 \frac{d K(\varsigma, \varsigma)}{d \varsigma}
$$

Taking the diagonal of $K(s, t)$ is the focusing operation (time-focusing) in 1-D. This can be seen from Eq. (2.10) that $K(\zeta, \zeta)$ is obtained from $R(2 \varsigma)$ after some multiple-scattering correction. $t=2 \varsigma$ is just the reflection travel-time of potential located at $\varsigma$. For the focusing of multiple-reflections, see the discussion of Rose's focusing operator in Section 2.5. The impedance parameter can be obtained from the scattering potential or from time-integral of $K(\zeta, t)$ (see Section 2.2).

From the definition in of scattering potential in (2.7), we see that the impedance function $\eta(\varsigma)$ needs to be continuous and twice-differentiable [25,34]. Although the Schrödinger equation (2.7) has an exact inverse solution for the potential based on the GLM (Gel'fand-Levitan-Marchenko) theory, this requirement of differentiability severely limits its applicability to seismic imaging/inversion where the reflected waves mostly coming from the reflections of sharp boundaries. In order to expand the applicability, we can expand the differentiability to include the Dirac type singularity. Let's decompose the scattering potential into a regular part and a singular part so we can have a mixed potential-impedance Schrödinger equation,

$$
\left(\frac{\partial^{2}}{\partial \varsigma^{2}}-\frac{\partial^{2}}{\partial t^{2}}\right) \psi(\varsigma, t)-q_{s i g}(\varsigma) \psi(\varsigma, t)-q_{r e g}(\zeta) \psi(\varsigma, t)=0,
$$

where $q_{\text {reg }}(\varsigma)$ is the regular part potential in which the $\eta(\varsigma)$ is smooth and twice- 
differentiable, and $q_{s i g}(\varsigma)$ is the singular part defined as

$$
q_{s i g}(\varsigma)=\left(\frac{1}{\eta(\varsigma)} \frac{\partial^{2}}{\partial \varsigma^{2}} \eta(\varsigma)\right)
$$

where $\eta(\varsigma)$ contains jumps (step function) and spikes (delta function). In fact we can derive the GLM integral equation from the mixed impedance-potential equation (2.12) which is also a Schrödinger type equation. To see this, we follow T. Tao's approach for derivation of time-domain GLM equation [35], Introduce the dual fundamental solution in time domain

$$
f(\zeta, t)=\delta(t-\varsigma)+K(\zeta, t) .
$$

For the dual solution, the role of time and space is reversed, so the causality is also reversed (dual causality). The Fourier transform of the dual solution is the Jost solution which is the frequency domain fundamental solution. Substitute (2.14) into Eq. (2.12), we can derive the GLM integral equation for the mixed impedance-potential equation (2.12) if the following condition is satisfied

$$
2 \frac{d}{d \varsigma} K(\zeta, \varsigma)=q_{r e g}(\varsigma)+\left(\frac{1}{\eta(\varsigma)} \frac{\partial^{2}}{\partial \varsigma^{2}} \eta(\varsigma)\right)
$$

This can be verified by substituting the above dual fundamental solution into Eq. (2.12). Since the $\delta$-function is a solution for the Schrödinger equation without potential, and the $[K(\varsigma, t)]$ operator is symmetric with respect to $\zeta$ and $t$ but has discontinuity along diagonal, the above result is easy to understand. Remember that in Eq. (2.15), $q_{\text {reg }}(\zeta)$ represents the smooth part of potential, which can generate reflections only for low-frequency incident waves; while in this paper we are mainly interested in strong scattering which represents the reflections by impedance jumps. Therefore we will deal only with the singular part $q_{\text {sig }}$ by dropping the regular potential $q_{\text {reg }}(\varsigma)$. In terms of scattering physics and seismic imaging reality, the smooth regular potential can produce only very weak signal for low-frequencies, while low-frequency seismic sources are usually not available, so the smooth-potential problem does not bear too much practical interest. On the contrary, scattering and inverse-scattering of singular potential, i.e. the potential representing impedance discontinuities, should attract both academic and practical interests. That is also to say that we will try to apply the GLM theory to solve the Schrödinger impedance equation (2.6) for the strong-scattering case.

In scattering and inverse-scattering problems of quantum physics and plasma physics, the task is to recover the potential from reflection measurement, so the standard approach is to solve first the GLM equation to get the K-matrix, and then apply differential operation for recovering the potential. From (2.7) we see that potential corresponds the second order derivative of impedance, so the potential recovery does not care the long-wavelength structure of impedance profile. From (2.11) we also see that the potential inversion takes use of only the high-wavenumber components of $K(\zeta, t)$. For 
impedance inversion we are interested in the recovery of low-wavenumber components of $K(\zeta, t)$.

From (2.7) and (2.11), K operator, potential $q_{\text {sig }}$ and impedance $\eta$ are related by

$$
q_{\mathrm{sig}}(\varsigma)=2 \frac{d}{d \varsigma} K(\zeta, \varsigma)=\left(\frac{1}{\eta(\varsigma)} \frac{\partial^{2}}{\partial \varsigma^{2}} \eta(\varsigma)\right)
$$

From the above equation, we can have two methods for the recovery of impedance $\eta(\varsigma)$. One is to solve a differential equation for $\eta(\varsigma)$ with the boundary conditions after solving the potential $q_{\text {sig }}$ which is used in Ware and Aki [25]. However, since $q_{\text {sig }}$ uses only the diagonal of the K-matrix, which resembles a layer-stripping method, the impedance recovery is unstable due to the singularity handling and error propagation problem. Let us look at the orders of singularity of $\eta, K$ and $q_{\text {sig }}$ for the case involving impedance jumps. To deal with parameter jumps, we define a unit step function $\Theta(x)$

$$
\Theta(x)=\left\{\begin{array}{c}
0, \text { for } x<0 \\
1 / 2, \text { for } x=0 \\
1, \text { for } x>0
\end{array}\right\}
$$

Then a discontinuity impedance profile can be expressed as

$$
\eta_{\text {sig }}(\varsigma)=\sum_{i=1}^{N} \Delta \eta_{i} \Theta\left(\varsigma-\varsigma_{i}\right)
$$

Then the differentiation of $\eta_{\text {sig }}(\varsigma)$ can be done symbolically,

$$
\frac{\partial}{\partial \varsigma} \eta_{\text {sig }}(\varsigma)=\sum_{i=1}^{N} \Delta \eta_{i} \delta\left(\varsigma-\varsigma_{i}\right), \quad \frac{\partial^{2}}{\partial \varsigma^{2}} \eta_{\text {sig }}(\varsigma)=\sum_{i=1}^{N} \Delta \eta_{i} \delta^{\prime}\left(\varsigma-\varsigma_{i}\right),
$$

leading to

$$
q_{\mathrm{sig}}(\varsigma)=\left[\sum_{i=1}^{N} \frac{\Delta \eta_{i}}{\eta_{i}} \delta^{\prime}\left(\varsigma-\varsigma_{i}\right)\right],
$$

where $\delta(\varsigma)$ and $\delta^{\prime}(\varsigma)$ are the unit delta function (the "unit impulse" ) and it's derivative (the "unit doublet"). From above equation, we see that the impedance function and Kmatrix involve the step function and delta function; while the potential $q_{\text {sig }}$ involves the derivative of delta function, and therefore has higher order singularities. In order to avoid singular operations involving $q_{s i g}$, we choose another approach to recover the impedance without involving the potential.

Since impedance profile involves only step function singularity, we can use the lowfrequency components of the K-matrix, namely the integration method, resulting in a stable inversion. We see that impedance also satisfies the following equation

$$
\frac{\partial^{2}}{\partial \varsigma^{2}} \eta(\varsigma)-q(\varsigma) \eta(\varsigma)=0
$$


As pointed out by Berryman and Greene [32], the above equation in fact is a zero-frequency Schrödinger equation, and the boundary conditions are $\eta(0)=1 / \sqrt{\left(\rho_{0} c_{0}\right)} ; \eta^{\prime}\left(0^{-}\right)=0$. The zero-frequency fundamental solution can be easily obtained by Fourier transforming equation (2.14) and setting $\omega=0$. Then the solution of Eq. (2.21) can be seen as

$$
\eta(\varsigma)=\eta(0)\left[1+\int_{-\varsigma}^{\varsigma} K(\varsigma, t) d t\right] .
$$

We see that in this way, the solution (2.22) for the impedance equation (2.6) not only keeps the low-frequency information in K-matrix, but also boosts the $l-f$ information in it by integration over $t$. After recovering $\eta(\varsigma)$, we can recover impedance from (2.5). In the case of known density, it is possible to reconstruct the velocity structure.

Now we further discuss the stability of impedance recovery based on (2.22), which can be also written as

$$
1+\int_{-\varsigma}^{\varsigma} K(\varsigma, t) d t=\frac{\eta(\varsigma)}{\eta(0)}=\frac{\sqrt{\rho_{0} \mathcal{C}_{0}}}{\sqrt{\rho_{\varsigma} \mathcal{C}_{\zeta}}}=\frac{\sqrt{Y_{\zeta}}}{\sqrt{Y_{0}}}
$$

where $Y=1 / \rho c$ is the admittance (reciprocal of impedance). For a $N$ layered medium, the above equation can be written as

$$
1+\int_{-\varsigma_{N}}^{\varsigma_{N}} K\left(\varsigma_{N}, t\right) d t=\frac{\eta\left(\varsigma_{N}\right)}{\eta(0)}=\frac{\sqrt{Y_{N}}}{\sqrt{Y_{N-1}}} \frac{\sqrt{Y_{N-1}}}{\sqrt{Y_{N-2}}} \cdots \frac{\sqrt{Y_{1}}}{\sqrt{Y_{0}}}=\frac{\sqrt{Y_{N}}}{\sqrt{Y_{0}}} .
$$

Form the above equation, we see that it is possible to recover the impedance (or admittance) layer by layer once you have resolved the potential, which is the usual layerstripping method and may suffer from the error-propagation problem. On the other hand, use the K-integral as shown by (2.23) can recover the impedance directly from $K(\zeta, t)$ and therefore avoids the error-propagation problem.

\subsection{Handling singularities in GLM method}

As we know that classic GLM theory requires the solution of Schrödinger equation to be continuous with a continuous derivative. However, when dealing with the impedance equation in seismic inversion, this requirement becomes a serious problem for practical applications (see Ware and Aki [25]). Think about the scattering physics. For reflection seismograms in one-dimensional acoustic media the most energetic reflection arrivals are from the discontinuities, while smooth variation of the media can produce only weak low-frequency reflections! Due to the frequency-band limitation of seismic sources, usually $l-f$ reflection signals are not observable. This renders the only useful reflection arrivals for inversions are those from discontinuities (interfaces, boundaries and faults). In this paper, I only discuss the discontinuity reflections for the purpose of impedance and velocity inversion and leave the mixed problems involving both continuous and discontinuous impedance changes for future research. 
To deal with discontinuity reflection, we need to treat the Dirac's delta function (or distributions) and it's derivative. Chadan and Sabatier [26, Section 17.4], introduced the "singular data function" to treat the mixed potential and impedance equation. Here we will concentrate on the impedance equation. The discontinuous impedance equation becomes a chain of Schrödinger equations with some continuity conditions at the boundaries. Using the singular data function, the equation system is transferred to a Schrödinger equation having continuous solution so the GLM theory can be applied. However, in geophysics, impedance inversion for layered media has been handled by the layer matrix approach with the aid of $z$-transform $[25,36,37]$. In this work, I will use the approximate Dirac delta function to handle the analysis and numerical calculations. From the theory of generalized function (or distribution), we can define the approximate delta functions as Gaussian, sampling function or other type smooth, differential functions. Here I adopt the Gaussian impulse, since it resembles a narrow delta impulse (see Korn and Korn [38]]):

$$
\delta(t, a)=\frac{a}{\pi} e^{-a^{2} t^{2}}, \quad \text { as } a \rightarrow \infty .
$$

In this way, the step function, representing impedance discontinuity, can be approximated by the integral of the Gaussian impulse, while the derivative of delta function (the doublet) can be approximated by $\delta^{\prime}(a, t)$. Then the GLM equation can be analytically or numerically implemented using this approximation. When the Gaussian impulse is narrow enough, we can use it as a symbolic unit delta function $\delta(t)$ satisfying

$$
\int_{a}^{b} f(\tau) \delta(\tau-t) d \tau=\left\{\begin{array}{c}
0, \text { if } \mathrm{t}<a \text { or } \mathrm{t}>b \\
f(t), \quad \text { if } a<t<b \\
\frac{1}{2} f(t), \text { if } t=a \text { or } t=b
\end{array}\right\} .
$$

In numerical simulations, if the source impulse is narrow enough and the reflection pulses do not have overlaps, then the approximate delta can be used in the numerical calculations, and the correct amplitude of the reflection pulses can be obtained by the integration over the pulse width. Now let us see what information is contained in $K(\zeta, t)$, especially in the diagonal elements of $K(\varsigma, \zeta)$. We need to understand the scattering physics involved in the mathematical operations. Fig. 1 shows a schematic diagram of the K operator. For our 1-D scattering problem, we chose the positive as downward for $z$-coordinate. $\varsigma(z)$ is the traveltime-equivalent $z$-coordinate after the Liouville transform. Seismic reflection data $R(t)$ are recorded on the surface $\varsigma=0$. The red line (diagonal of the right-lower quadrant) marks the diagonal line of the K-operator $K(\zeta, \zeta)$. Since $K(\zeta, t)$ is a triangle matrix, satisfying $K(\zeta, t),-\varsigma \leq t \leq \varsigma$, so the solution of $K(\zeta, t)$ (satisfying the Schrödinger equation) only exist in the purple shaded area. $K(\zeta, \zeta)$ can be looked as the first-arrival event-line in space-time $(\zeta, t)$, similar to Einstein's "light-cone". The first reflection signals can be observed along the event-line $K(\varsigma, \zeta)$ (the location of reflectors). Therefore, the yellow triangle on the right (with the horizontal-axis as its bisect) is the area of causal solution, and any events of multiple scattering (later arrivals) can be seen in this area, with $R(t)$ as an example (observed on the surface). We can say that the yellow 


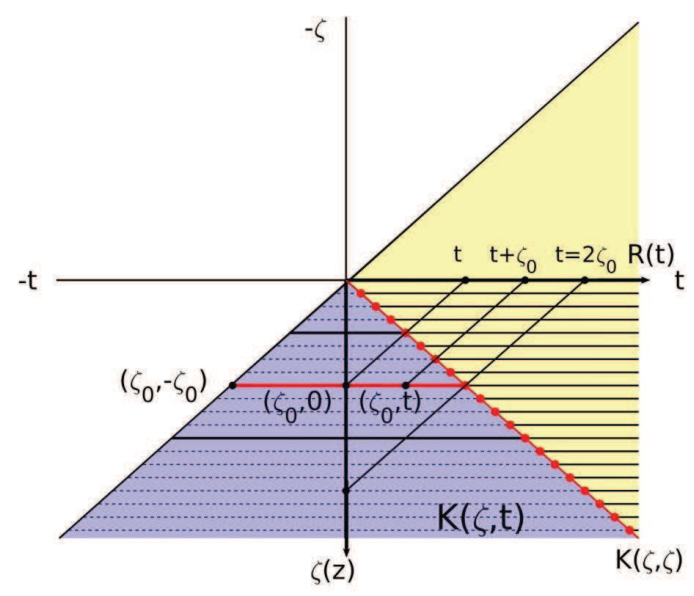

Figure 1: Schematic diagram of the $\mathrm{K}$ operator and its region of definition (purple area).

area is the "future area". On the contrary, or complementarily, the purple area is the "past area", where the events in the past are recorded. In the future area, the event is seen by forward propagation; while in the past area, the events can be met by back-propagation (reverse-time). Since $K(\zeta, t)$ is a dual fundamental solution, dual causality (the role of time and space is reversed) is observed. We know $K(\zeta, \zeta)$ is the line of first-reflection arrivals, the past record $K(\zeta, t)$ is a single-reflection record which has been solved by the GLM equation. This is consistent with Snieder's analysis that only the Born data (single reflection data) contribute to the recovery of the scattering potential. Here we show that it is also only the single-reflection data contributing to the impedance recovery (Eq. (2.22)). Note that since the Schrödinger equation in our context is second order in time, so the Born solution includes also the anti-causal single-reflections. This becomes clearly seen in the following numerical examples.

\subsection{Liouville transform, the forward-scattering renormalization and velocity inversion}

First, we prove that the Liouville transform eliminates the forward scattering, so there is only backscattering in action for Schrödinger impedance equation.

In the process of reducing the wave equation to the Schrödinger impedance equation, we perform change of both independent and dependent variables. The dependent variable change is to have a energy-flux normalized field variable so that the scattering matrix becomes unitary and therefore the impedance can be recovered by reflection data alone. The independent variable change from $z$ to $s$ is by the Liouville transform which incorporates the velocity change into the traveltime converted $s$. In this one-dimensional scattering problem, this corresponds to a forward-scattering renormalization. The Born series divergence has been well-known and thoroughly studied in quantum field theory and 
solved by the renormalization theory/method (see, e.g., Delamotte [39]). In exploration geophysics, the multiple-forescattering single-backscattering (MFSB) approximation or the De Wolf approximation (DWA) has been introduced as a renormalization solution to the divergent Born series (for a review, see e.g. [24,40]. The main idea is to split the scattering operator into forward scattering and backscattering parts and substitute it into the Born series. Then we can have all combinations of higher order forward and backward scattering terms. The De Wolf approximation $[8,9,41,42]$ corresponds to neglecting multiple backscattering (reverberations), i.e. dropping all the terms containing two or more backscattering terms but keeping all the multiple forward scattering terms in the series. Then sum up all the multiple forward-scattering terms resulting in a mass operator which includes all the nonlinear interactions of the point perturbations on the forward path. We know that the Born series divergence is mainly caused by the coherent summation of forward scattering responses from each point along the path such that the scattering amplitude increases infinitely in the forward direction (infra-red catastrophe). The renormalization procedure solves the divergence problem. It has been proved that by a renormalization procedure based on the renormalization group (RG) theory, the forward scattering effect can be transformed into phase accumulation (integration) along the forward propagation path [43]. In 1-D problem, the split of forward and backward scattering is easy to do, so the Liouville transform

$$
\varsigma(z)= \begin{cases}z / c_{0}, & \text { for } z \leq 0 \\ \int_{0}^{\varsigma} d z / c(z), & \text { for } z \geq 0\end{cases}
$$

which incorporates the forward scattering effect (phase variation due to velocity change) into the traveltime variable, so the resulted Schrödinger equation can only have backscattering (quantified by reflection and transmission coefficients) effect. We may make a remark here that transmission is also a backscattering effect. Due to backscattering, incident wave loses energy, and the transmitted wave is only incident field subtracting the reflected energy.

\subsection{GLM solution, focusing operator, and multiple-scattering removal}

Next we explain the multiple-reflection removal of the GLM equation proved by Snieder [44] and it's relation with focusing operator of J. Rose [45]. In the paper, Snieder pointed out and proved the following. If you expand the scattering response $R(t)$ into a Born series from forward modeling of the Schrödinger equation, and in the same way expand $K(x, t)$ into a Taylor series for Eq. (2.10), then it was shown that only the Born data (first term of the Taylor series) contributes to the reconstruction of the potential, and all the higher order terms in the data do not contribute. It means that the nonlinear components in the data, i.e. the multiple-scattering effects in the data, are eliminated by solving the GLM integral equation: the second-order Born term is being cancelled by an internal interaction (second order scattering) of the first order scattering; the higher order terms are cancelled by the interaction of the all the lower-order terms (multiple-scattering). In the end of the process (de-scattering process), there left only the first order term (Born data) 
contributing to the potential recovery. Since the potential recovery is from the diagonal elements of $\mathrm{K}$ matrix, we can consider $K(s, t)$ as composed of only the single-reflected waves (including both casual and anticausal waves). Therefore, by using the GLM solution $K(\zeta, t)$, potential and impedance inversions become linear operations. The demonstration by Snieder [44] has profound implication to the theory and method of waveform inversion. In higher dimensions (2D or 3D), similar strategy should hold.

Now I will use the concept of J. Rose's focusing source or focusing operator [45, 46] to further explain that the GLM equation can focus the wave to the selected point $\varsigma$ by picking the first-reflection arrival $R(t+\varsigma)$ at the time $t+\varsigma$ on the surface and removing all the multiple-reflections (the tail) through solving the GLM equation. "Focusing source" means you set up a specific "incident wave" (a source time-function) for a specified point at depth $\varsigma_{0}$, then the wavefield propagate and focus only at $\varsigma_{0}$ at $t=0$. The focusing source do not have any radiation to other points than $\varsigma_{0}$ due to mutual cancellation by interference. The "focusing operator" means an operator applying to the reflection data (impulse response) yields focused image at subsurface scattering sources (scattering potential).

The focusing source function (the incident wave needed for the focusing) is defined by Rose [45] as

$$
f\left(\varsigma_{0}, t-\tau\right)=\delta\left(t-\tau+\varsigma_{0}\right)+K\left(\varsigma_{0}, t-\tau\right)
$$

Here $\varsigma_{0}$ is the point to focus and $\tau$ is the corresponding time-shift for the focusing. In fact, this is also the dual fundamental solution (see Eq. (2.14)). When we apply this source to the Schrödinger equation (2.7) as the source term (primary source), the wavefield radiated by this source will focus on $\varsigma_{0}$ and vanishes at all other locations. The delta function (wavefront) of the above source function is to put the pulse into the correct location (the focus); the long tail of the source function, $K\left(\varsigma_{0}, t\right),\left(-\varsigma_{0} \leq t \leq \varsigma_{0}\right)$, is to cancel all other reflection signals, including primary and multiple reflections by scattering potential above depth $\varsigma_{0}$. This magic "tail" is proved to be the solution of GLM equation (or Marchenko equation) [45]. From this we can see that $K\left(\varsigma_{0}, t\right)$ has an important role for multiple removal. Now we discuss the focusing operator $F(\zeta, t)$ with the focusing source time-function $f\left(\varsigma_{0}, t-\tau\right)=f\left(\varsigma_{0}, t-\varsigma\right)$ as its kernel (remembering that the wave speed is 1 in GLM theory). What will happen when we apply the operator to the reflection data $R(t)$, namely the impulse response measured on the surface? It will focus the reflection pulses in the data back to the scattering sources in the medium. It can be explained as the follows. It is known that the focusing operator is the complex-conjugate or time-reversal of the point-response function which generates the reflection seismogram from a point scatterer in the known medium. The measured impulse response is just the linear superposition of the point-response function of all the point scatterers in the medium. Therefore, applying the focusing operator to the impulse response of the medium yields an image field or generalized holographic field (GHF) $h(\varsigma, t-\varsigma)$ inside the medium [47-49]. Invoking the imaging principle by coinciding the local incidence and reflection, we obtain the scatterer's image $h(\zeta, \zeta)$, which is a collection of all the scattering points. In operator form it 
reads

$$
\mathbf{h}=\mathbf{F R}=(\mathbf{I}+\mathbf{K}) \mathbf{R},
$$

where $\mathbf{I}$ is the identity matrix, $\mathbf{F}$ is the focusing operator, and $\mathbf{h}, \mathbf{R}$ are vectors. Substitute the kernel (2.28) into the operator and write into explicit form, yielding

$$
h(\varsigma, t)=R(t+\varsigma)+\int_{-\varsigma}^{\varsigma} K(\varsigma, \tau) R(t+\tau) d \tau .
$$

Rose [45] has proved that the image field $h(\zeta, t)=-K(\zeta, t)$. So the tail of the focusing source function can be found by solving the GLM equation (or Marchenko equation).

From the above analysis, we see that $K(\zeta, t)$ can be considered as the trailing part of a focusing operator which is the solution of GLM equation. It is interesting to look at the other aspect of $K(\zeta, t)$, as a holographic image field of the reflectors. $K(\zeta, t)$ contains the Born refection data extracted from the real seismogram. Of course, this "data" set is not an actually recorded casual records. It is composed of both casual and anti-casual Born solutions to the Schrödinger equation. This view is consistent with the above explanation as a holographic field.

According to Snieder [44, equation 15], the $n$th order coefficient of Taylor series $K_{n}$ of $K(\zeta, t)$ can be related to the corresponding multiple-scattering series $R_{n}$ of surface data $R(t)$. However, we may replace the surface reflection data with the scattered field in the whole medium $U(s, t)$, then we have the two perturbation series

$$
K=\sum_{n=1}^{\infty} \varepsilon^{n} K_{n}, \quad U=\sum_{n=1}^{\infty} \varepsilon^{n} U_{n}
$$

where $\varepsilon$ is a smallness parameter and $U=U^{+}+U^{-}$with $U^{+}$and $U^{-}$as the casual and anti-casual parts of the scattered field, respectively. The surface data is related to the scattered field $U(\zeta, t)$ through the time-shift relation $R(t)=U^{+}(0, t)$ and $R(t+\varsigma)=U^{+}(\zeta, t)$ where $U^{+}(\zeta, t)$ is the casual part of the scattered field. With this replacement, we repeat Snieder's derivation, resulting in

$$
\begin{aligned}
K_{1} & =-U_{1}, \\
K_{2} & =-U_{2}+U_{1} U_{1}, \\
K_{3} & =-U_{3}+U_{2} U_{1}+U_{1} U_{2}-U_{1} U_{1} U_{1}, \\
& \ldots \\
K_{n} & =-U_{n}+\sum_{m=1}^{n-1}(-1)^{m} \sum_{i_{1}+\cdots+i_{m}=n-1} U_{i_{1}} \cdots U_{i_{m}} .
\end{aligned}
$$

The above equation means that the potential is completely determined by the first Born data $K_{1}=-U_{1}$, the higher order reflections (higher order coefficients of the Born series to the Schrödinger equation) have no contributions to the image $K(\zeta, \zeta)$ due to the 
mutual cancellations as shown in (2.32). This is consistent with the discussion on focusing source function and the focusing operator. The kernel (focusing source function) of the focusing operator removes all multiples produced by the potential above the focusing point, then the focusing operator will remove all the multiples during the recursive process in solving the GLM equation. Therefore, only the Born data (single reflection data) make real contribution in the final stage of impedance inversion in GLM approach. Remember that the single-reflection data $K_{1}(s, t)$ have both casual and anti-casual events.

The single-reflection data in $K(\zeta, t)$ is the basis of potential recovery by differentiation (2.16) and the impedance recovery by integration (2.22). Impedance recovery is more important in seismology. The scattered field $U$ in Schrödinger equation in fact is like reflected energy-flux when interacting with potential or impedance. The flux is focused at the focal depth, and beneath the focal depth, the flux splits into casual and anti-casual components. Due to the anti-symmetry $U^{-}=-U^{+}$, the integration of all the defocused scattered fields becomes null, and only the flux at the focal point has net contribution to the impedance recovery (2.22).

\subsection{Recovery of velocity structure: inverse Liouville transform and de-renormalization}

From reflection data we recovered the impedance jumps at different traveltime points. For a constant density medium or if we know the density distribution, in principle we can recover the velocity structure layer-by-layer by an inverse Liouville transform (time to depth conversion)

$$
z(\varsigma)=z_{0}+\int_{0}^{\varsigma} c(\tau) d \tau
$$

At each upper face of a jump, you derive the velocity jump from the impedance inversion (with known density), then you know the velocity at the lower face of jump and the spatial location of the next jump. Of course this layer-stripping method have the error propagation problem and there is no other information to stabilize the inversion in the 1-D case. However, in the 2-D and 3-D cases, the situation can be improved, since the velocity structure can be checked by a time-space focusing operator (remember that in 1-D case, there is only time focusing, and no spatial focusing available). If the velocity structure is correct, the focusing of the next impedance jump will be perfect so an optimization algorithm can be set up to get the best estimate of velocity or the boundary location. These techniques are the focusing analysis and velocity analysis in seismic exploration (see, e.g., Stolt and Bensen [50]). This is also the reason why seismic direct envelope inversion (DEI) can successfully invert for velocity structure of layered media by a two-dimensional acquisition geometry (see next section).

As we stated in the previous section that the Liouville transform is a forward scattering renormalization, then this inverse Liouville transform is a reverse renormalization or de-renormalization. Renormalization changes the Born volume scattering into a traveltime perturbation along the path. In the 1-D case, this is done recursively slab-by-slab. In the 
de-renormalization process, the traveltime change is changed back to slab scattering. In the case of constant velocity-jump, it is changed to the thickness variation, so the location of next jump can be determined. However, in the 1-D case, there is no spatial focusing, and the time-focusing is only a traveltime pickup. For this reason, there is no method to stabilize the layer stripping inversion in the 1-D case. Nevertheless, the principle of inverse Liouville transform can be applied to the high-dimensional strong-scattering inversion as we will demonstrate in the next section.

\subsection{Numerical tests on the impedance and velocity inversion by GLM method}

To demonstrate acoustic impedance inversion using GLM theory, we use a simple 1-D acoustic model of a slab with $\rho=1, c=2$ embedded in a homogeneous background of $\rho=1, c=1$ (Fig. 2a). For forward modeling, we use the propagator matrix method (see, e.g., Aki and Richards [34, Chapter 12]) to produce the frequency domain seismogram and then transform to the time domain. The singularities are handled by the approximate delta functions as illustrated in previous section. The time domain reflection seismogram is plot in Fig. 2b.

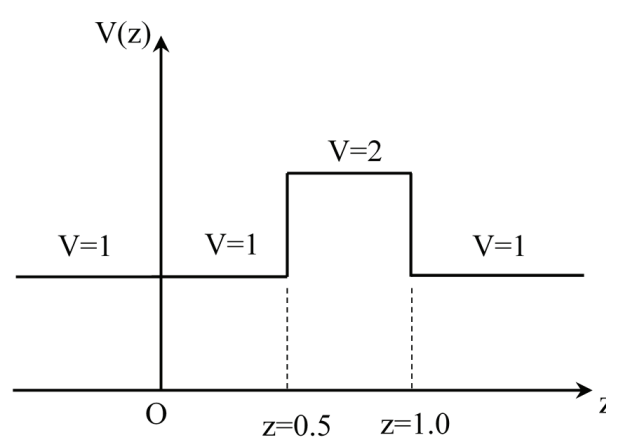

(a)

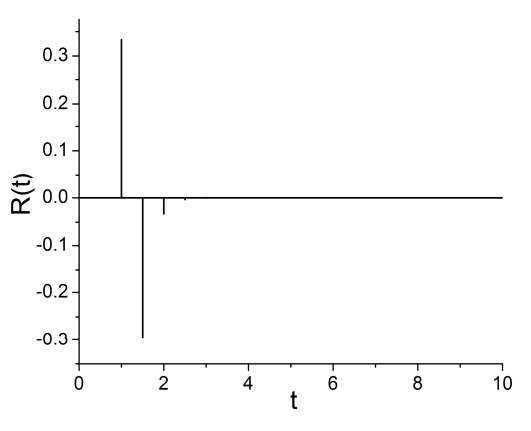

(b)

Figure 2: One-dimensional impedance model (left) and the reflection seismogram (right).

After solving the GLM integral equation by an iterative method [33], the final solution of $K(\zeta, t)$ is plot in Fig. 3, and the time-profiles at different $\varsigma$ locations of $K(\zeta, t)$ are shown in Fig. 4. We see that at the locations above and below the slab boundaries, the integration of the $K(s, t)$ along time is zero, and only within the slab the integration gives a finite value corresponding to the impedance. The result of impedance recovery by using (2.22) is shown in Fig. 5a. By inverse Liouville transform with known density $\rho$, we can recover the velocity $c$ distribution as shown in Fig. $5 \mathrm{~b}$. As we pointed out already, the inverse Liouville transform has a layer-stripping procedure and may have some error accumulation problem which needs further investigation. 


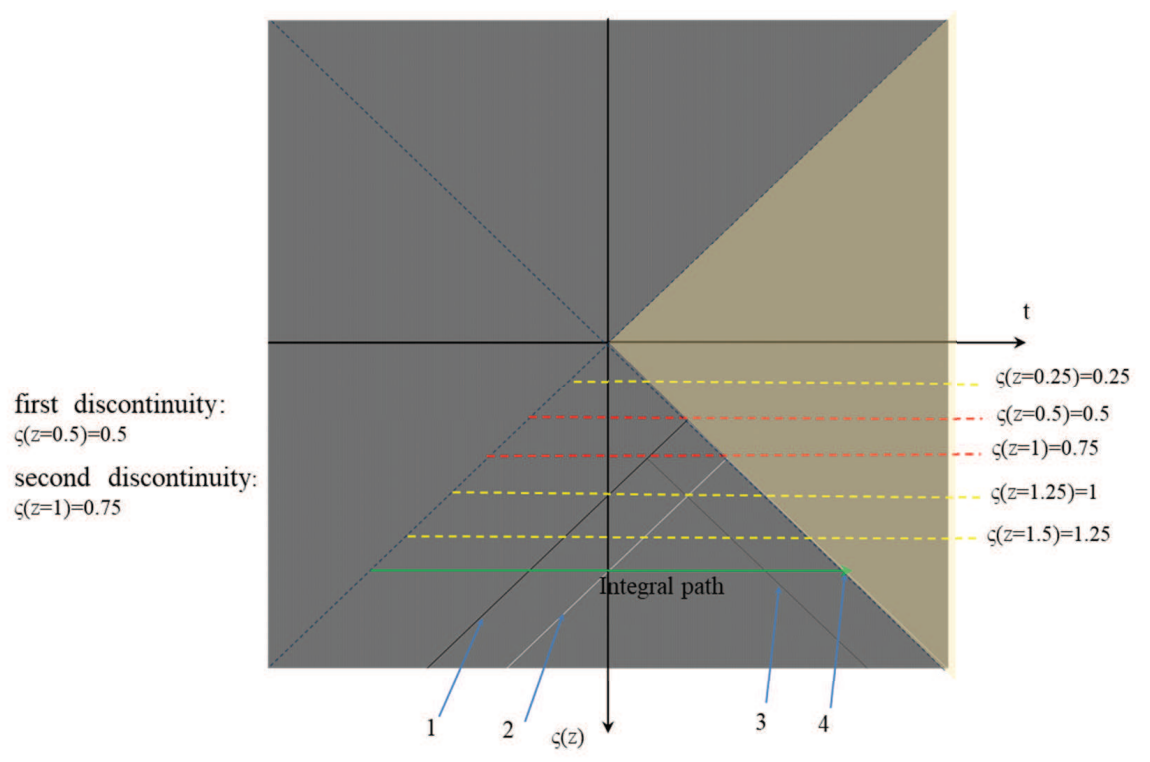

Figure 3: Final solution of $K(\zeta, t)$ by solving GLM integral equation.

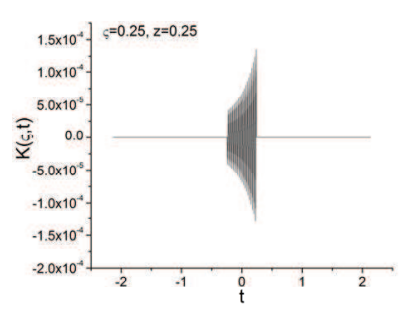

(a)

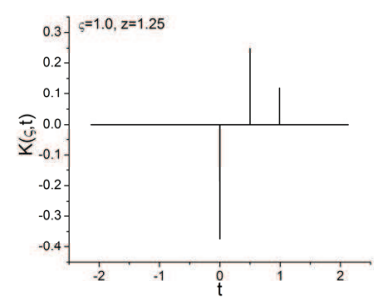

(d)

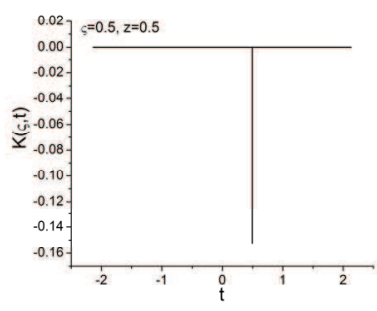

(b)

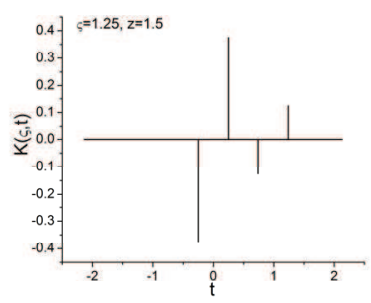

(e)

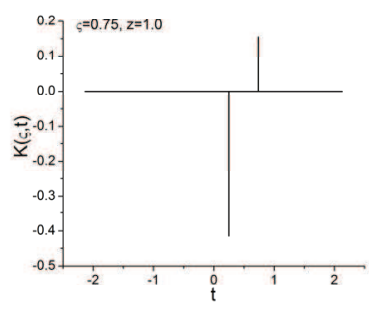

(c)

Figure 4: $K(\zeta, t)$ as time-series for different location $\varsigma$. The two boundaries of the slab are at $\varsigma=0.5(z=0.5)$ and $\zeta=0.75(z=1.0)$. 

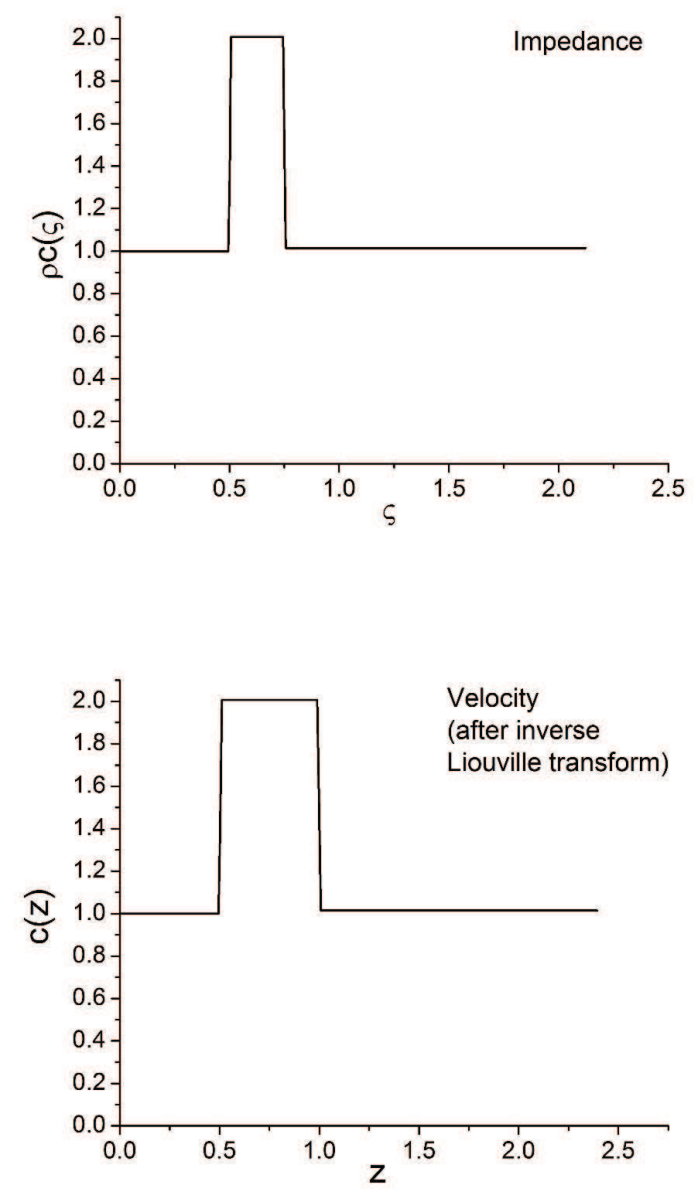

(b)

Figure 5: Impedance $(\rho c)$ and velocity $(c)$ recovery of the model in Fig. 2.

\section{Strong-scattering inversion in 3-D by DEI (Direct Envelope Inversion)}

In general, the inverse-scattering process based on wave equation is complicated and delicate. It is because that the scattering process in complex media is a strongly nonlinear with respect to the parameter changes. The process involves strong interference between the Born scattering and multiple scatterings. Such as for boundary reflection, strong constructive interference forms a sharp reflection pulse; meanwhile strong destructive interference diminishes all the single-scattering contributions from volume elements inside the boundary. Inverse-scattering process tries to reverse the scattering process, such as to regenerate all the already destroyed waves to restore the Born data from the tangled 
nonlinear data. This is doomed to be not an easy job. It is like the resurrection of dead waves. This is the reason why IBS for wave equation can easily diverge or fall into wrong solutions.

Seismic envelope inversion has been introduced and developed to recover the longwavelength background velocity structure without relying on the ultra-low frequency source $[13,16,17,19,24]$. Traditional envelope inversion was still based on the weakscattering theory and borrowed the wavefield Fréchet derivative from the waveform inversion for the indirect envelope inversion. Therefore, the traditional approach is not applicable to the strong-nonlinear case such as the inversion involved with salt structures.

Recently, multi-scale direct envelope inversion (MSDEI) has been proposed $[18-21,51]$ to tackle the difficult task of strong-scattering inversion. The envelope operator (or demodulation operator) transforms waveform data into envelope data and conduct a nonlinear filtering in the envelope domain. Removing of multiple forward-scattering effect seems much easier in dealing with envelope data, since there is no interference. In the early stage of development, energy scattering is taken as the physical model for envelope data without polarity [18-21,51]. In order to compensate the lack of polarity information, a joint misfit functional for envelope data plus the conventional waveform data is adopted to recover the large-scale sharp-contrast structure. The recent introduction of the signed envelope data obtained by the signed demodulation operator [51-53] leads to a more compatible envelope data for the theory/method of DEI (direct envelope inversion). It will be shown in this paper that the signed envelope trace is in fact a filtered version of the reflection time-series in GLM theory for inverse Schrödinger scattering. In this section, I first point out the problem of using the indirect sensitivity operator in the traditional envelope inversion in the strong-scattering case, Then I derive the Kirchhoff integral representation for the strong-scattering case and show its equivalence to the Schrödinger-like system of equations. This will pave the way to connect DEI to the GLM theory of solving the inverse Schrödinger scattering.

\subsection{The problem in linearization of envelope inversion using indirect sensitivity operator for strong-scattering case}

In waveform inversion, the waveform sensitivity operator (Fréchet derivative) can be expressed in an operator form

$$
\delta \mathbf{p}=\mathbf{F}_{p} \delta \mathbf{v}=\frac{\partial \mathbf{p}}{\partial \mathbf{v}} \delta \mathbf{v}=\mathbf{G}_{0} \mathbf{Q}_{0} \delta \mathbf{v},
$$

where $\delta \mathbf{p}$ (vector) is the wavefield variation due to velocity perturbation $\delta \mathbf{v}$ (vector), $\mathbf{F}_{p}$ is the waveform Fréchet derivative (operator), $\mathbf{G}_{0}$ is the background Green's operator, and $\mathbf{Q}_{0}$ is the virtual source operator (VSO), which transforms $\delta \mathbf{v}$ into an equivalent source (scattering source) field. From the above definition, we can see that the Fréchet derivative is a linearized sensitivity operator which is only valid when the velocity perturbation is small and therefore the scattered wavefield is also small. Similar to above equation for 
the waveform inversion, we can formulate the envelope sensitivity operator (ESO) in a form

$$
\delta \mathbf{e}=\mathbf{F}_{E} \delta \mathbf{v}=\frac{\partial \mathbf{e}}{\partial \mathbf{v}} \delta \mathbf{v}=\mathbf{G}_{E} \mathbf{Q}_{E} \delta \mathbf{v},
$$

where $\mathbf{F}_{E}$ is the envelope sensitivity derivative, $\mathbf{G}_{E}$ is the envelope Green's operator, and $\mathbf{Q}_{E}=\mathbf{Q}_{0}^{(e)}$ is the envelope VSO. In this way, we derive the envelope sensitivity operator (Fréchet derivative) directly based on envelope scattering theory, and therefore no weak scattering assumption or weak nonlinearity of waveform sensitivity operator is imposed. However traditionally envelope Fréchet derivative (indirect envelope sensitivity operator) is derived from a chain rule of functional derivatives, and the implementation is relied on the use of waveform Fréchet derivative (WFD). In operator form, the indirect envelope Fréchet derivative $(\partial \mathbf{e} / \partial \mathbf{v})_{\text {indir }}$ can be written as

$$
\left(\frac{\partial \mathbf{e}}{\partial \mathbf{v}}\right)_{\text {indir }} \simeq \frac{\partial \mathbf{e}}{\partial \mathbf{p}} \frac{\partial \mathbf{p}}{\partial \mathbf{v}}=\frac{\partial \mathbf{e}}{\partial \mathbf{p}} \mathbf{F}_{p} .
$$

The chain rule of differentiation is in fact making linearization to both the nonlinear partial derivatives $(\partial \mathbf{e} / \partial \mathbf{p})_{N L}$ and $\mathbf{F}_{p}^{N L}=(\partial \mathbf{p} / \partial \mathbf{v})_{N L}$, that may destroy the advantage of the new data functional $\mathbf{F}_{E}=\partial \mathbf{e} / \partial \mathbf{v}$ which has better linear relationship between data and pa-

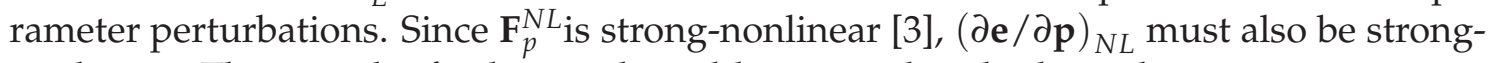
nonlinear. This may be further explained by expanding both nonlinear operators into Taylor series around the points of interests:

$$
\begin{aligned}
& \left(\frac{\delta \mathbf{e}}{\delta \mathbf{p}}\right)_{N L}=\frac{\delta \mathbf{e}}{\delta \mathbf{p}}+\frac{1}{2 !} \frac{\delta^{2} \mathbf{e}}{\delta \mathbf{p}^{2}} \delta \mathbf{p}+\cdots, \\
& \left(\frac{\delta \mathbf{p}}{\delta \mathbf{v}}\right)_{N L}=\frac{\delta \mathbf{p}}{\delta \mathbf{v}}+\frac{1}{2 !} \frac{\delta^{2} \mathbf{p}}{\delta \mathbf{v}^{2}} \delta \mathbf{v}+\cdots .
\end{aligned}
$$

Then the indirect envelope sensitivity operator becomes

$$
\left(\frac{\delta \mathbf{e}}{\delta \mathbf{v}}\right)_{\text {indir }}=\left(\frac{\delta \mathbf{e}}{\delta \mathbf{p}}\right)_{N L}\left(\frac{\delta \mathbf{p}}{\delta \mathbf{v}}\right)_{N L}=\frac{\delta \mathbf{e}}{\delta \mathbf{p}} \frac{\delta \mathbf{p}}{\delta \mathbf{v}}+\frac{1}{2 !}\left[\frac{\delta \mathbf{e}}{\delta \mathbf{p}} \frac{\delta^{2} \mathbf{p}}{\delta \mathbf{v}^{2}} \delta \mathbf{v}+\frac{\delta^{2} \mathbf{e}}{\delta \mathbf{p}^{2}} \delta \mathbf{p} \frac{\delta \mathbf{p}}{\delta \mathbf{v}}\right]+\cdots .
$$

Taking only the first order term, it becomes the chain rule of differentiation which is a double linearization and only valid for weak nonlinearity. In the case of strong nonlinearity, the nonlinear functional derivative (sensitivity operator) becomes model dependent. Furthermore, for strong-scattering case, the nonlinearity may be so strong such that the Taylor series becomes divergent and the linear approximation becomes meaningless. On the other hand, the direct envelope sensitivity operator has a direct linear relationship to the impedance contrast for boundary scattering. This is the physical basis for impedance inversion using inverse Schrödinger scattering by solving the GLM equation. The GLM equation is to remove the effect of multiple-reflections. As we showed in Section 2.5, the recovery of potential or impedance by GLM solution uses only the single-scattered 
data (Born data of Schrödinger scattering). Although DEI do not remove the multiplereflections, the better linear relationship of envelope to impedance jump is a major advantage of DEI.

\subsection{From wave equation to Schrödinger-like equation in 3D for strong- scattering case}

To use direct envelope Fréchet derivative means to linearize directly the envelope variation due to medium perturbation without relying on functional derivative. We know that in large strong-contrast media, such as salt structures, the strong reflection signals are from boundary scattering which can be considered as frequency-independent. This is the feature of potential scattering or impedance scattering in Schrödinger equation. Now we derive the integral representation of boundary scattering and show that the corresponding differential equation is a Schrödinger-type equation. I will compare the common feature of envelope inversion with the GLM inverse-scattering for the 1-D Schrödinger equation and discuss their differences.

For the sake of simplicity, we deal with only the scalar wave equation to demonstrate the principle. First we express the wave equation in an operator form

$$
L_{0} \delta \mathbf{p}=-\left(L-L_{0}\right) \mathbf{p}=\mathbf{Q} \mathbf{p},
$$

where

$$
\begin{aligned}
& L=\left[\nabla^{2}-\frac{1}{v^{2}(\mathbf{x})} \frac{\partial^{2}}{\partial t^{2}}\right], \\
& L_{0}=\left[\nabla^{2}-\frac{1}{v_{0}^{2}(\mathbf{x})} \frac{\partial^{2}}{\partial t^{2}}\right],
\end{aligned}
$$

and $Q$ is the scattering potential operator for the wave equation with its kernel

$$
Q\left(\mathbf{x}, \mathbf{x}^{\prime}, t\right)=\left[\frac{1}{v^{2}(\mathbf{x})}-\frac{1}{v_{0}^{2}(\mathbf{x})}\right] \frac{\partial^{2}}{\partial t^{2}} \delta\left(\mathbf{x}-\mathbf{x}^{\prime}\right) .
$$

We see that the scattering potential for wave equation is time-dependent, so in the frequency-domain, frequency-dependent. As we discussed for the 1-D case, renormalization process for strong scattering can change the potential into time-independent. From the partial differential equation (3.6), we can derive the corresponding integral equation, Lippmann-Schwinger equation (LSE),

$$
\delta \mathbf{p}=L_{0}^{-1} \mathbf{Q} \mathbf{p}=\mathbf{G}_{0} \mathbf{Q} \mathbf{p},
$$

where $\mathbf{G}_{0}$ is the Green's operator.

Now let us treat the strong scattering case. Scattered waves from boundary scattering can be modeled as a summation of reflected waves from all the sharp boundaries inside 
the considered volume. Assume $\Omega$ as the collection of boundaries surrounding different domains, $\Gamma \equiv\left\{\Gamma_{n}\right\}, n=1,2, \cdots, N$. Each domain has smooth velocity variation inside the domain. For any curved boundary (interface) $\Gamma_{1}$, the scattered waves can be formulated by a representation integral (Kirchhoff integral),

$$
\delta p(\mathbf{x})=-\int_{\Gamma_{1}}\left\{p_{s}\left(\mathbf{x}_{0}\right) \nabla_{n} G_{F}\left(\mathbf{x} ; \mathbf{x}_{0}\right)-G_{F}\left(\mathbf{x} ; \mathbf{x}_{0}\right) \nabla_{n} p_{s}\left(\mathbf{x}_{0}\right)\right\} d s\left(\mathbf{x}_{0}\right),
$$

where $G_{F}$ is forward-scattering approximated Green's function in the exterior region (for smooth exterior media, $\left.G_{F}=G_{0}\right)$, $d s\left(\mathbf{x}_{0}\right)$ is a boundary element at $\mathbf{x}_{0}$ on the boundary $\Gamma_{1}, p_{s}\left(\mathbf{x}_{0}\right)$ is the scattered field at that point, $\hat{\mathbf{n}}$ is the local outward normal and $\nabla_{n}$ is the normal gradient at that point. $p_{s}\left(\mathbf{x}_{0}\right)$ can be obtained by solving the boundary integral equation (BIE). We know that the scattered wave on the outer boundary is proportional to the local impedance contrast. In this paper, we further simplify the relation to the case of small-angle reflections. In modern seismic exploration with full coverage of shot array and large-aperture receiver array for each shots, data redundancy is true for most of the cases. In DEI (direct envelope inversion) or inverse Schrödinger scattering, we use only the reflection signals and better linearity is expected between reflection coefficients and impedance jumps for small-angle reflections. Hence limiting to small-angle reflections is reasonable. By this approximation, the scattered field $p_{s}\left(\mathbf{x}_{0}\right)$ on the boundary for a delta-pulse source can be approximated as

$$
p_{s}\left(\mathbf{x}_{0}\right)=\gamma\left(\mathbf{x}_{0}, \hat{\mathbf{n}}\right) p_{0}\left(\mathbf{x}_{0}\right)=\gamma\left(\mathbf{x}_{0}, \hat{\mathbf{n}}\right) G_{0}\left(\mathbf{x}_{0}, \mathbf{x}_{s}\right),
$$

where $x_{s}$ is the source location, $\gamma\left(\mathbf{x}_{0}, \hat{\mathbf{n}}\right)$ is a factor dependent on the local impedancecontrast and the incident angle. Considering that $\nabla_{n} G_{F}\left(\mathbf{x}_{0} ; \mathbf{x}_{s}\right) \doteq-\nabla_{n} G_{F}\left(\mathbf{x}_{g} ; \mathbf{x}_{0}\right)$ for small reflection angles, where $x_{g}$ is the receiver location, we derive the approximation of (3.10),

$$
\delta p\left(\mathbf{x}_{g}, \mathbf{x}_{s}\right) \simeq 2 \int_{\Gamma_{1}} G_{F}\left(\mathbf{x}_{g} ; \mathbf{x}_{0}\right) \nabla_{n} \gamma\left(\mathbf{x}_{0}, \hat{\mathbf{n}}\right) G_{F}\left(\mathbf{x}_{0} ; \mathbf{x}_{s}\right) d s\left(\mathbf{x}_{0}\right) .
$$

In order to be consistent with the volume integral formulation, we may change the surface integral into volume integral by assuming zero-reflection outside all the real boundaries $\Gamma \equiv\left\{\Gamma_{n}\right\}$. For acoustic media, reflection coefficient by a plane boundary is

$$
\gamma=\frac{Y_{1}^{(n)}-Y_{2}^{(n)}}{Y_{1}^{(n)}+Y_{2}^{(n)}}=\frac{Z_{2}^{(n)}-Z_{1}^{(n)}}{Z_{2}^{(n)}+Z_{1}^{(n)}},
$$

where $Z^{(n)}$ is the normal component of acoustic impedance, and $Y^{(n)}$ is that of admittance (inverse of impedance), and

$$
Y^{(n)}=\frac{1}{\rho c} \cos \theta, \quad Z^{(n)}=\frac{\rho c}{\cos \theta}
$$


where $\theta$ is the reflection angel. For small angle reflection $(\cos \theta \simeq 1)$, we have

$$
\gamma \approx \frac{Z_{2}-Z_{1}}{Z_{2}+Z_{1}}=\frac{Y_{1}-Y_{2}}{Y_{2}+Y_{1}}=\frac{-\Delta Y}{2 Y_{0}+\Delta Y^{\prime}}
$$

where $Y_{0}=Y_{1}$ and $\Delta Y=Y_{2}-Y_{1}$. Since we discuss only small-angle reflections, $Y$ or $Z$ will be same as its normal component. In this way, the admittance jump can be related to the reflection coefficient,

$$
\Delta Y=\frac{-2 \gamma}{1+\gamma}
$$

In this way, the reflection singular function is related to the admittance jump (similar to (2.18) and (2.19) for the 1-D case). By handling the singularities, (3.12) can be written into a volume integral,

$$
\delta p\left(\mathbf{x}_{g}, \mathbf{x}_{s}\right) \doteq 2 \int_{V} G_{F}\left(\mathbf{x}_{g} ; \mathbf{x}_{0}\right) \nabla_{n}\left[\gamma_{\Gamma}(\mathbf{x}) G_{F}\left(\mathbf{x} ; \mathbf{x}_{s}\right)\right] d v(\mathbf{x}),
$$

where $\gamma_{\Gamma}(\mathbf{x})$ exists only on the boundary $\Gamma$, defined as

$$
\gamma_{\Gamma}(\mathbf{x}) \triangleq \gamma(\mathbf{x}, \hat{n}(\mathbf{x})) \delta\left(\mathbf{x}-\mathbf{x}_{0}\right), \quad \mathbf{x} \in V ; \quad\left(\mathbf{x}_{0}, \hat{n}\right) \in \Gamma,
$$

and the volume element $d v(\mathbf{x})$ depends on the local surface normal since it is converted from the surface integral (3.12). Since we use the small-angle reflection assumption and the boundary reflection physics, $\delta p\left(\mathbf{x}_{g}, \mathbf{x}_{s}\right)$ in above equation in fact is scattering amplitude, which is proportional to the energy-flux (with sign or polarity).

Here we make some remarks on the Kirchhoff integral representation for scattered field from boundary scattering (reflection). From (3.10) and (3.12), we see that the scattered field from boundary scattering is frequency-independent in contrast to the volume scattering derived from wave equation. In order to use the concept of local reflection coefficient (3.11), the boundary should be smooth compared with the wavelength, so the Kirchhoff representation in fact is a high-frequency approximation. This is consistent with the analysis for Schrödinger equation for acoustic impedance scattering (see the discussion around Eq. (2.8)). In the Kirchhoff integral (3.17), $G_{F}\left(\mathbf{x}_{0} ; \mathbf{x}_{S}\right)$ is the incident field, so $\gamma_{\Gamma}(\mathbf{x})$ plays the role of scattering potential (singular potential). Therefore, Eq. (3.12) or (3.17), and the related system of boundary integral equations can be viewed as a system of Schrödinger equations. Kirchhoff approximation is also called physical-optics approximation [54], which means propagates like waves but interacts with boundary like rays (geometric-optics approximation). This is similar to the duality of quantum field in Schrödinger equation. This also explains the difference between the energy-scattering formulation [18-21,51] and the latest signed envelope (envelope with polarity) inversion based on energy-flux formulation $[25,52,53,55])$. Although good results have been obtained by this new approach in salt structure inversion, this paper is the first paper in trying to establish the physical-mathematical foundation of DEI with signed envelopes. Even though some approximation is involved, the transfer from wave equation to Schrödinger-like equation system has paved the way to treat strong-scattering inversion with methods radically different from the weak-scattering based methods. 


\subsection{Envelope data and reflection time-series}

Now let us discuss the link between the solution of Schrödinger equation and the envelope data in envelope inversion, especially in direct envelope inversion. Here comes the second important attribute of strong-scattering inversion: sparse reflections. From scattering theory, these two attributes, frequency-independence and sparse reflection are the consequences of the same strong-scattering physics. If a slab is thick enough compared with the dominate wavelength (strong-scattering), then pulse-split ("cycle-skipping) must happen, resulting in sparse reflections. I have pointed out that Schrödinger equation or the corresponding integral equation form has a h-f asymptotic solution. In fact, Schrödinger scattering is better behaved for high-frequencies. For broad-band cases, the reflected wave $\delta p\left(\mathbf{x}_{g}, \mathbf{x}_{s}\right)$ in time-domain is consisted of marrow pulses. In the limiting case, the pulses approach delta-functions, which is similar to the data used in the GLM theory. Using a delta pulse as source, the reflection records become $R(t)$, a reflection time-series. However, in seismic reflection experiments, we do not have delta-pulse as source. Due to the limited bandwidth of our acquisition system, the seismograms become oscillating traces, consisting of a series of wavelets. Normally this kind of seismograms cannot be converted to a series of reflection spikes due to the strong interference between different wavelets. However, for the case of strong-scattering, the reflection spikes should be "sparse" and therefore satisfy the sparsity requirements of separability (see the mathematical theory of super-resolution by Candes and Fernandez-Granda [56]. After the reflection time-series is reconstructed from the received waveform data, such as by a convex optimization algorithm, the theory and method of solving schrodinger equation can be applied to the strong-scattering inversion (inverse-scattering). In envelope inversion, instead, an envelope operator, such as the demodulation operator based on Hilbert transform, is used to obtain the envelope traces (envelograms). For sparse reflections, which has the same criterion for strong-scattering, the individual reflection wavelets do not have strong interference such that they can be separated into different events, each has a recoverable envelope. In demodulation theory, this means the envelope signal and the carrier signal satisfy the Bedroisin-Brown theory of separability. As a result, demodulation operator transforms the waveform trace into an envelope trace. The latter is similar to a series of approximate delta pulses (envelogram). The polarity of reflection can be obtained from the instantaneous phase in Hilbert transform based moethd [22, 52,57]. In this way, the envelope traces can be treated as approximate solutions of the corresponding Schrödinger equation.

We understand that the equation involved is not an exact 3D Schrödinger equation, since the above equation is only an exterior equation (such as in the exterior region of salt domes), and $\gamma_{\Gamma}(\mathbf{x}, \hat{n})$ has to be solved by a coupled equation system for both exterior and interior (inside salt domes) equations, and the interior equation has different Green's function from the exterior problem. The other difference from the 1-D Schrödinger potential equation is that the Liouville transform is no longer available in the 3-D case, so the knowledge of the internal velocity structure becomes another task. 

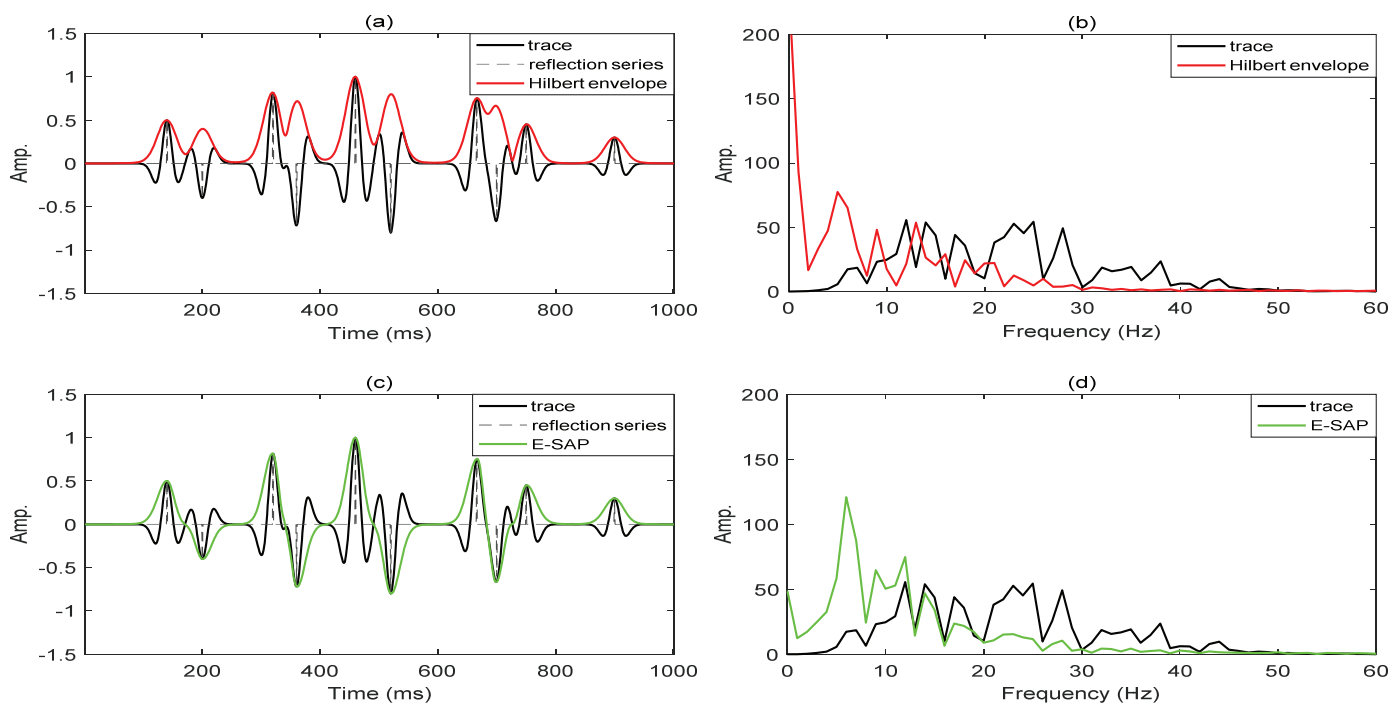

Figure 6: Comparison of envelope data with and without polarity. The synthetic trace is generated with 10 reflectors convolved with a $20 \mathrm{~Hz}$ Ricker wavelet. (a) is the Hilbert envelope (the red line), and (c) is the polarity-bearing envelope (green curve). The dotted spikes are the reflection time-series, the black curve is the original waveform data; (b) and (d) are the corresponding spectra.

Nevertheless, since the potential does not depend on frequency, it has many features of Schrödinger impedance equation. So we can borrow many techniques from the 1-D inverse Schrödinger scattering to the theory / method of 3-D envelope inversion.

In the following we demonstrate the new feature of signed envelope (envelope with polarity) with an example.

Standard envelope data are obtained by taking instantaneous amplitude of the analytical signal derived from the waveform data through analytical transform based on the Hilbert transform (we will refer it as "Hilbert envelope") [13,58]. However, the Hilbert envelope does not have the polarity information. It is possible to put the polarity information into the envelope data, so that the polarity-bearing envelope (or simply the signed envelope) will have much resemblance to the reflection time-series. There are two approaches in combining the polarity into the envelope data. One is to use the signed demodulation method $[22,55,57]$, the other is the smoothed apparent polarity method [52]. One example of comparison between Hilbert envelope and signed envelope is shown in Fig. 6. It can be seen that the signed envelope in fact is a filtered version of the reflection series in Schrödinger scattering. Comparing the spectra of Hilbert envelope and the signed envelope (Fig. $6 \mathrm{~b}$ and $6 \mathrm{~d}$ ), we see that the signed envelope keeps the useful ULF (frequencies below the source frequency band) unchanged, but removes most of the near-zero frequency components, and therefore reduces substantially the artifacts by the near-zero frequencies. Due to the strong similarity of envelope inversion to the 1-D Schrödinger impedance inversion, especially when the signed envelope is used, the 
GLM theory and method will certainly shed some light for future development of direct envelope inversion.

\subsection{Direct envelope inversion (DEI)}

With the direct envelope Fréchet derivative (sensitivity operator), we can directly backpropagate (refocusing) the envelope residual to the model space without losing too much low-frequency information of the envelope data. To recover the long-wavelength (or low$\mathrm{K})$ velocity structure from the envelope data, we perform a nonlinear filtering in the data domain. The multi-scale direct envelope inversion (MSDEI) [18-22,59] or the signed envelope inversion $[52,53,55]$ take this approach. The nonlinear operator is referred to the envelope data reduction from the waveform data, since the Hilbert envelope is nonlinear to the waveform data. After the Hilbert envelope data or the signed envelope data are obtained, a linear filter is applied to the envelope data to extrapolate the data to get the ULF (ultra-low-frequency) component for recovering the long-wavelength background velocity structure. Fig. 7 gives an example of the signed envelope data from the SEG 2D salt model (see Fig. 9a) shown by the green curve. Fig. 8 shows the frequency spectra of different types of envelope data compared with the waveform data for seismic traces from SEG salt model. The red line is for the signed demodulated (SD) envelope, and the pink and green lines are for the low-passed SD envelopes. We see that the low-passed $\mathrm{SD}$ envelopes have much lower frequencies. These low-frequency components will be backpropagated (re-focused) to become the long-wavelength structure of the salt bodies. The data domain low-K recovery for envelope data is much simpler that the nonlinear operation for the waveform data or for the waveform inverse sensitivity operator. To say this in mathematical language, we use the nonlinear implicit function theorem as follows.

From (3.2), we derive the nonlinear envelope SO (sensitivity operator),

$$
\left(\frac{\delta \mathbf{e}}{\delta \mathbf{v}}\right)_{N L}=\left(\frac{\delta \mathbf{e}}{\delta \mathbf{p}}\right)_{N L}\left(\frac{\delta \mathbf{p}}{\delta \mathbf{v}}\right)_{N L}^{\prime}
$$

where $\mathbf{e}$ is the envelope data, and $\mathbf{p}$ is the waveform data. The subscript "NL" means "nonlinear". This equation is a generalization of the chain rule of differentiation. From the above relation, we can derive

$$
\left(\frac{\delta \mathbf{p}}{\delta \mathbf{v}}\right)_{N L}=\left(\frac{\delta \mathbf{e}}{\delta \mathbf{p}}\right)_{N L}^{-1}\left(\frac{\delta \mathbf{e}}{\delta \mathbf{v}}\right)_{N L}^{\prime}
$$

where $\left(\frac{\delta \mathbf{e}}{\delta \mathbf{p}}\right)_{N L}^{-1}$ is the inverse envelope operator. The above equation is the implicit functional theorem for nonlinear case [43]. Then we obtain the waveform inverse sensitivity operator

$$
\left(\frac{\delta \mathbf{p}}{\delta \mathbf{v}}\right)_{N L}^{-1}=\left(\frac{\delta \mathbf{e}}{\delta \mathbf{v}}\right)_{N L}^{-1}\left(\frac{\delta \mathbf{e}}{\delta \mathbf{p}}\right)_{N L} .
$$




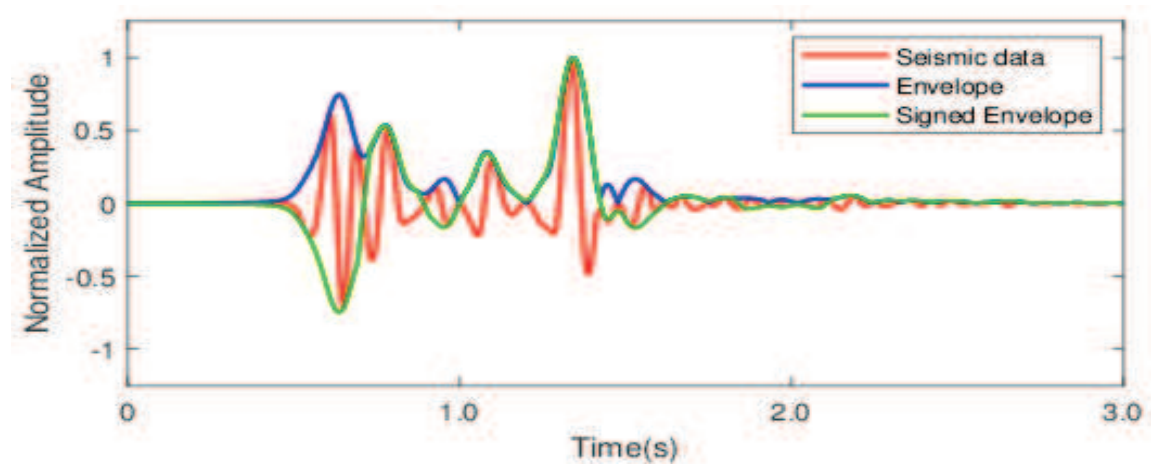

Figure 7: The signed envelope data are similar to filtered reflection time-series; One trace of data from the SEG salt model. (waveform data: red, Hilbert envelope data: blue; and signed envelope data: green)

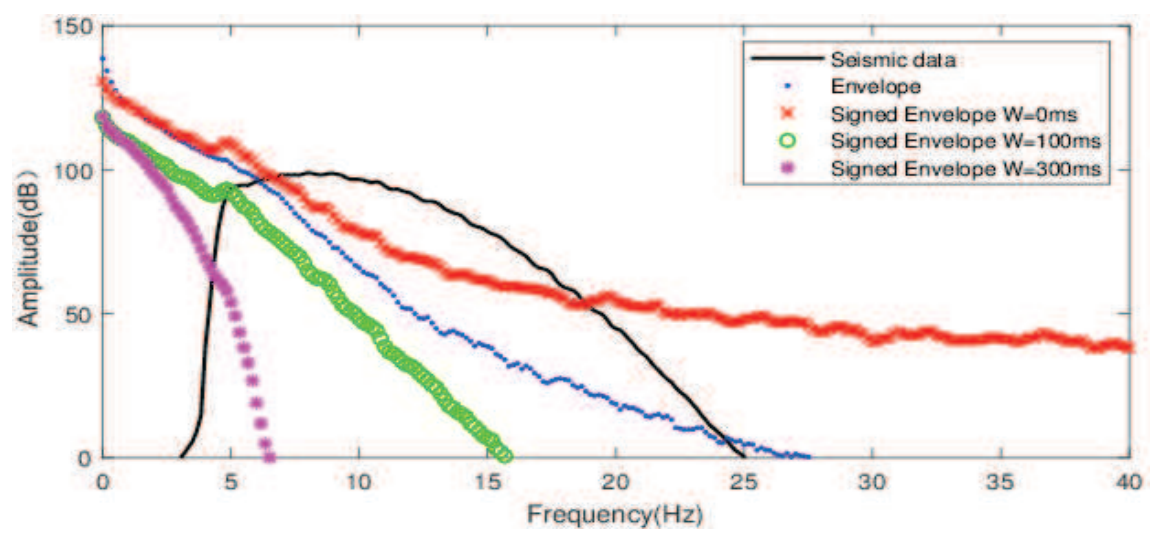

Figure 8: Spectra of envelope data with singed demodulation: Black line is for the waveform data, blue is for Hilbert envelope, red, green and pink are for the signed envelopes with different low-pass filters.

As we discussed in Section 3.1, $(\delta \mathbf{e} / \delta \mathbf{v})_{N L}$ has much weaker nonlinearity than the waveform sensitivity operator, which we mark as $(\delta \mathbf{e} / \delta \mathbf{v})_{w N L}$, where "wNL" denotes weak nonlinear. On the other hand, we mark strong nonlinear as "sNL". Then the above equation can be rewritten as

$$
\mathbf{F}_{p}^{-1} \equiv\left(\frac{\delta \mathbf{p}}{\delta \mathbf{v}}\right)_{s N L}^{-1}=\left(\frac{\delta \mathbf{e}}{\delta \mathbf{v}}\right)_{w N L}^{-1}\left(\frac{\delta \mathbf{e}}{\delta \mathbf{p}}\right)_{s N L} .
$$

In terms of functional derivative, the above equation shows that an inverse strongnonlinear functional derivative (inverse waveform sensitivity kernel) can be implemented via a weak-nonlinear inverse functional derivative (inverse envelope sensitivity kernel) applying to a nonlinearly transformed data set based on the nonlinear implicit theorem. In gradient method, we use the adjoint operator to substitute the inverse oper- 

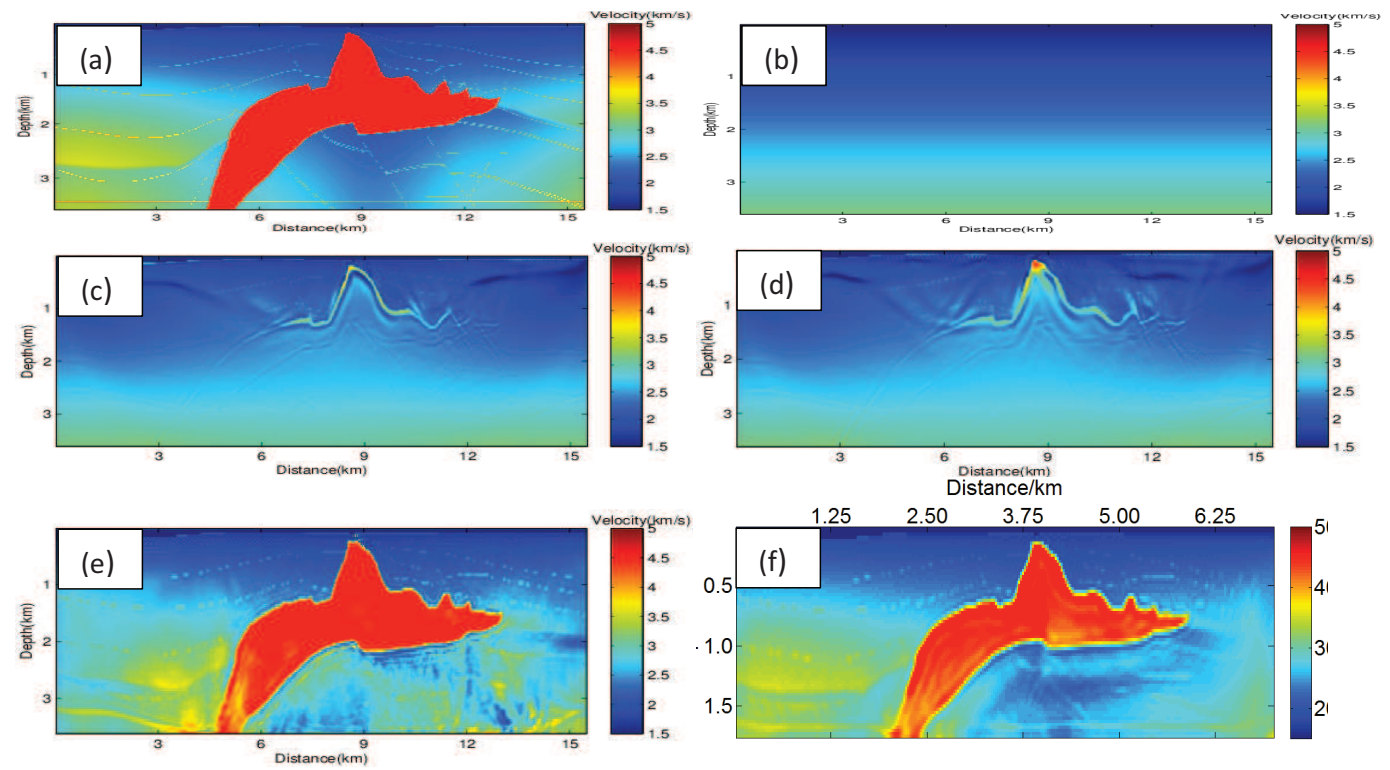

Figure 9: MS-DEI using envelope data with polarity (envelope by signed demodulation) for SEG/EAGE 2D salt model: a) True model, b) Initial model, c) Result of conventional FWI, d) Result of envelope inversion using indirect sensitivity operator derived from chain rule, e) MS-DEI result using direct envelope sensitivity operator using envelope data by signed-demodulation. f) MS-DEI result by direct envelope sensitivity operator using envelope phase-data for polarity information.

ator for weak-nonlinear operator. Therefore, linear-filtering the envelope data is equivalent to applying a strong-nonlinear inverse sensitivity operator to the nonlinear waveform data. Even though the filtering procedure in envelope data domain and the inverse nonlinear sensitivity operation applying to the waveform data have same final goal, the filtering procedure on envelope data is much easier due to absence of interference.

We know that the strongest nonlinearity for FWI exists in the recovery of largescale strong-contrast structure (the low-wavenumber components). Therefore the lowwavenumber recovery can be accomplished by DEI using a nonlinear data functional, the envelope function. After recovering the low-wavenumber component of velocity structure, the rest of inversion becomes weak-nonlinear and can be carried out by conventional FWI. Fig. 9 shows the results of envelope inversion (for large-scale structure) plus conventional FWI (to add high-wavenumber component to the inversion) for the SEG salt model. We use a Ricker wavelet with a dominant frequency of $9 \mathrm{~Hz}$ as source in the test (cut from $4 \mathrm{~Hz}$ below). Fig. 9a is the true model, and Fig. $9 \mathrm{~b}$ is the initial model for inversion. Fig. 9c is the result of conventional FWI, and Fig. $9 \mathrm{~d}$ is the result using conventional envelope-inversion (using the indirect sensitivity operator derived by the chain rule). The last two figures (Figure 9e and 9f) are the results by Direct envelope inversion (DEI). Fig. 9e is MS-DEI using signed envelopes [22,52,55]; Fig. 9f is MS-DEI by a joint phase-amplitude inversion through time-frequency analysis [53]. We see that 
DEI using the signed envelope can obtain good recovery of the salt structure (Figs. 9e, 9f). The DEI method using envelope-phase with normalized envelope-amplitude can enhance the signal from deep structure reflections, so the inversion has better recovery of deep structures (Fig. 9f). Some results have also been obtained for source-independent MS-DEI [23].

\section{Discussion and conclusion}

After analyzing the GLM theory and the direct envelope inversion (DEI) (especially the multi-scale DEI), I point out the similarity and differences between the two approaches. These methods belong to a category of strong-scattering inversion or inverse Schrödinger scattering (inversion problem for Schrödinger equation). This method untangles the strong nonlinearity to remove the multiple-scattering effect from the data so that the transformed data will have better linearity to the model parameter change. However, the traditional GLM theory/method deals mostly on potential inversion which is a highwavenumber inversion and is quite different from the impedance inversion and velocity inversion in acoustics and seismology. In this work, following the work in seismic literature on layered media, summarized in the book of Aki and Richards (Volume II) and the work by Sabatier (in Chadan ad Sabatier [26]), I introduce the singular function for impedance jumps and then apply the GLM theory to impedance and velocity inversion, especially to the long-wavelength structure recovery by solving the GLM equation.

When we look at the three-dimensional impedance inversion problem, things become quite different from the case of one-dimensional. Although the beautiful GLM theory for 1-D inverse scattering emerged a long time ago, 3-D exact inverse-scattering solution is still in the developing stage (see the books by Newton [60], Chadan and Sabatier [26], Pike and Sabatier [61]). As pointed out by Roger Newton in the preface of his book [60], the data (observations) are 5-dimensional (two incident angles, two receiving angles, one frequency or time), but the potential is only 3-dimensional. To figure out a one-to-one correspondence between observables and potential parameters becomes much more complicated. This "large degree of over-determination in higher-dimensional inverse scattering problems", is the major source of difficulty in finding exact solutions; On the other hand, this may turn out to be very favorable for optimization based inversion methods. More data redundancy, more stable the solution will be by minimizing the misfit-functional of observations versus predictions. From this point of view, the direct envelope inversion (DEI) tries to incorporate the advantages of both approaches into the direct envelope inversion for strong-scattering inversion. The derivation, examples and discussions in this paper may serve as the first attempt in this direction, and many questions and problems remain to be answered and solved in future researches.

For three-dimensional scattering problems, there is another significant difference between quantum scattering and seismic scattering (classical wave scattering). Schrödinger equation is devised by Schrödinger to model the wave-particle duality in quantum me- 
chanics. When propagating, the wave function of particles such as photons, behaves like waves; however, when interacting with atoms, nucleus, and molecules, behaves like particles. This is similar to the physical optics approximation or Kirchhoff approximation in optics: propagating as waves but reflecting by sharp boundary like particles. This is why we can borrow some techniques from inverse Schrödinger scattering for envelope inversion. Nevertheless, there is significant differences in the application goals. As stated in R. Newton's book, when applying the inverse Schrödinger scattering to atoms and nucleus, the potentials to be solved mainly are central potentials. Only for molecular scattering, non-central potentials have to be involved. On the contrary, in 3-D seismic impedance inversion, inclusions are irregular, sharp-contrast bodies, central potential inversion seems have very little applications. For irregular boundaries of strong-contrast inclusion, the impedance jumps have more dimensions than three, since the normal vectors of boundary elements $(\hat{n})$ need another two dimensions to specify. This demonstrate the difference between the potential inversion and the impedance inversion.

Direct envelope inversion (DEI) combines some features from GLM theory and the Newton's local optimization algorithm for the estimate of focusing operator in the velocity inversion, therefore opened a new approach for 3-D strong-scattering inversion. An example for the SEG/EAGE salt model demonstrates the validity of direct envelope inversion. The success of direct envelope inversion raises also some interests to understand its link to the 3-D inverse Schrödinger scattering problem. It is also conceivable that the combination of strong-scattering inversion (inverse Schrödinger scattering) and weak-scattering inversion (local optimization based inversion) may create some inversion methods working for a whole range of inversion problems in geophysical exploration.

\section{Acknowledgments}

I am grateful to Yingcai Zheng, Morten Jakobsen, Chuck Mosher, Huijing He, Guoxin Chen, Yong $\mathrm{Hu}$, Shaohuan $\mathrm{Zu}$, Bo Feng and Benfeng Wang for helpful discussions. Special thank is to Huijing He for his calculation of the 1-D GLM solution examples. 2-D direct envelope inversion results are provided by Guoxin Chen, Yong Hu and Yuqing Wang from their thesis works. This work is supported by WTOPI-Inv (Wavelet Transform On Propagation and Imaging/Inversion for seismic exploration) Research Consortium and other funding resources at the Modeling and Imaging Laboratory, University of California, Santa Cruz. We are grateful to our Consortium sponsors for their financial support and allowing us to publish our research results.

\section{References}

[1] Tarantola, A., 2005. Inverse Problem Theory and Methods for Model Parameter Estimation, Society for Industrial and Applied Mathematics, Philadelphia, PA.

[2] Virieux, J. and Operto S., 2009. An overview of full-waveform inversion in exploration geophysics, Geophysics, 74(6), wcc1-wcc26. 
[3] Wu, R.S. and Zheng, Y., 2014, Nonlinear partialderivative and its De Wolf approximation for nonlinear seismic inversion, Geophys. J. Int., 196, 1827-1843.

[4] Prosser, R.T., 1976, Formal solutions of inverse scattering problems. II, J. Math. Phys., 17, 1775-1779.

[5] Kouri, D.J. and A. Vijay, 2003, Inverse scattering theory: Renormalization of the LippmannSchwinger equation for acoustic scattering in one dimension, Physical Review E, 67, 740014$1-12$.

[6] Yao, J., A.C. Lesage, B. G. Bodmann, F. Hussain, and D. J. Kouri, 2014, One dimensional acoustic direct nonlinear inversion using the Volterra inverse scattering series, Inverse Problems, 30, 075006 (17pp).

[7] Weglein, A., F. V. Araujo, P.M. Carvalho, R. H. Stolt, K. H. Matson, R. T. Coates, D. Corrigan, D. J. Foster, S. A. Shaw and H. Zhang, 2003, Inverse scattering series and seismic exploration, Inverse Problems, 19, R27-R83.

[8] De Wolf, D.A. 1985, Renormalization of EM fields in application to large-angle scattering from randomly continuous media and sparse particle distribution, IEEE Trans. Ant. and Propag. AP-33, 608-615.

[9] Wu, R.S., 2003, Wave propagation, scattering and imaging using dual-domain one-way and one-return propagators, Pure and Appl. Geophys., 160(3/4), 509-539.

[10] Wu, R.S., B. Wang and C. Hu, 2015, Inverse thin-slab propagator in T-matrix formalism based on nonlinear sensitivity kernel for wave-equation tomography, Inverse Problems, 31, 11,115004 .

[11] Jakobsen, M. and R.S. Wu, 2016, Renormalized scattering series for frequency domain waveform modelling of strong velocity contrasts, Geophys. J. International, 206, 880-899.

[12] Geng, Y., W. Pan, K. A. Innanen, 2018, Frequency-domain full-waveform inversion with non-linear descent directions, Geophys. J. International, 213, 739-756.

[13] Bozdag, E., J. Trampert, and J. Tromp, 2011, Misfit functions for full wave-form inversion based on instantaneous phase and envelope measurements, Geophys. J. Int., 185, 845870.

[14] Wu, R.S., J. Luo, B. Wu, 2013, Ultra-low-frequency information in seismic data and envelope inversion: in Proceedings of the SEG Technical Program, Expanded Abstracts, 3078-3082.

[15] Wu R. S., Luo J.R. and Wu B. 2014. Seismic envelope inversion and modulation signal model. Geophysics 79, WA13WA24.

[16] Chi, B., Dong, L. and Liu, Y., 2014. Full waveform inversion method using envelope objective function without low frequency data, J. Appl. Geophys.,109, 3646.

[17] Luo, J. and $\mathrm{Wu}, \mathrm{R} ., 2015$, Seismic envelope inversion: reduction of local minima and noise resistance. Geophysical Prospecting, 63: 597-614

[18] Wu, R.S and Chen, G. X. 2017a. Multi-scale seismic envelope inversion for strong-nonlinear FWI, 2017 CGS/SEG International Geophysical Conference, Qingdao, China, 17-20.

[19] Wu R.S and Chen G. X. 2017b. New Fréchet derivative for envelope data and multi-scale envelope inversion. In 79th EAGE conference and Exhibition 2017. Tu A3 12.

[20] Wu R.S and Chen G. X. 2017c, Multi-scale seismic envelope inversion for salt structures using a new direct envelope Fréchet derivative, SEG Expanded Abstract.

[21] Chen, G., R.S. Wu, Y. and S. Chen, 2017, The nonlinear data functional and Multi-scale seismic envelope inversion: Algorithm and methodology for application to salt structure inversion, SEG Expanded Abstract.

[22] Chen, G., R.S. Wu, Y. Wang, and S. Chen, 2018a, Multi-scale direct envelope inversion using signed demodulation, CPS/SEG (Beijing) Expanded Abstract.

[23] Zhang, P., R.S. Wu and L. Han, 2018, Source-independent seismic envelope inversion based 
on the direct envelope Fréchet derivative, Geophysics, in press.

[24] Wu, R.S., 2018, Untangle the nonlinearity in strong-nonlinear FWI: De-scattering, renormalization and de-interference, CPS/SEG (Beijing) Expanded Abstract.

[25] Ware J.A and Aki, K. 1969, Continuous and discrete inverse scattering problems in a stratified elastic medium. I: planes at normal incidence. J Acoust Soc Am. 45(4):911-921.

[26] Chadan, K. and P.C. Sabatier, 1989, Inverse problems in Quantum scattering theory, second edition", Springer-Verlag

[27] Gel'fand, I.M. and B. M. Levitan, 1951, On the determination of a differential equation from its spectral function, Amer. Math. Soc. Transl. (1955), 1, pp. 253-304.

[28] Marchenko, V.A., 1955, The construction of the potential energy from the phases of the scattered waves, Sokl. Adad. Nauk. SSSR 104, 695-698. [Math Rev. 17, 740 (1956)].

[29] Newton, R.G., 1982, Scattering theory of waves and particles, second ed., Dover.

[30] Chew, W.C., 1990, Waves and fields in inhomogeneous media, Van Norstrand Reinhold.

[31] Kay I, 1955, The inverse scattering problem New York University Research Report no EM-74.

[32] Berryman, J.G. and R. R. Greene, 1980, Discrete inverse methods for elastic waves in layered media, Geophysics. vol. 15, no. 2, 213-233.

[33] Ge, D. B., 1987 An iterative technique in one-dimensional profile inversion, Inverse Problems 3 399-406

[34] Aki, K. and Richards P., 1980, Quantitative Seismology, vol. II, Chapter 12 "Inverse problems in seismology", W.H. Freeman and Company.

[35] Tao, T., Israel-Gelfand: 1. Inverse scattering, http:/ / terrytao.wordpress.com/2009/10/07//israel-gelfand/

[36] Goupillaud, P., 1961, An approach to inverse filtering of near-surface layer effects from seismic records, Geophysics, v. 26, p. 754-760.

[37] Claerbout, J. F., 1968, Synthesis of a layered medium from its acoustic transmission response: Geophysics, v. 33, 264-269.

[38] Korn, G.A. and T.M. Korn, 1961, Mathematical handbook For Scientists and Engineers, Dover.

[39] Delamotte, B., 2004, A hint of renormalization, Am. J. Phys., 72, 170-184.

[40] Wu, R.S., Xie, X.B. and Wu, X.Y., 2007, One-way and one-return approximations for fast elastic wave modeling in complex media, Chapter 5 of " Advances in Wave Propagation in Heterogeneous Earth", edited by R.S. Wu and V. Maupin (V.48 of " Advances in Geophysics", Series editor R. Dmowska), Elsevier, 266-323.

[41] De Wolf, D.A. 1971, Electromagnetic reflection from an extended turbulent medium: Cumulative Forward-scatter single-backscatter approximation, IEEE Trans. Ant. and Propag. AP-19, 254-262.

[42] Wu, R.S., 1994, Wide-angle elastic wave one-way propagation in heterogeneous media and an elastic wave complex-screen method, J. Geophys. Res., 99, 751-766.

[43] Kirkinis, E., 2008a, Renormalization group interpretation of the Born and Rytov approximations, J. Opt. Soc. Am. A, 2499-2508.

[44] Snieder, R. The role of the Born approximation in nonlinear inversion, 1990, Inverse Problems, 6, 247-266.

[45] Rose J. H., 2001, Single-sided focusing of the time-dependent Schrödinger equation Phys. Rev. A 65012707.

[46] Aktosun, T. and J. H. Rose, 2002, Wave focusing on the line, J. of Mathematical Physics 43(7), 3717-3745.

[47] Porter, R.P., 1970, Diffraction-limited scalar image formation with holograms of arbitrary 
shape., J. Opt., Soc., Am. 60, 1051-1059.

[48] Bojarski, N., 1983, Generalized reaction principles and reciprocity theorems for the wave equation and the rekationship between the time-advanced and time-retarded fields, $\mathrm{J}$. Acoust. Soc. Am., 74(1), 281-285.

[49] Langenberg, K.J., 2002, Linear scalar inverse scattering, in "Scattering: Scattering and inversion scattering in pure and applied science", Pike and Sabatier, eds. Academic Press, 121-141.

[50] Stolt and Benson, 1986, Seismic migration, theory and practice, Geophysical Press, Ltd.

[51] Chen, Guoxin, Ru-Shan Wu and Shengchang Chen, 2018c, Reflection Multi-Scale Envelope Inversion, Geophysical Prospecting, https:/ / doi.org/10.1111/1365-2478.12624.

[52] Wang, Y., R.S. Wu, Guoxin Chen, and Zhenming Peng, 2018, Seismic modulation model and envelope inversion with smoothed apparent polarity, Journal of Geophysics and Engineering, in press, https:/ /doi.org/10.1088/1742-2140/aac54d

[53] Hu, Y., R.S. Wu,L.G. Han, P. Zhang, 2018, Multi-scale energy weighted envelope-phase inversion using direct envelope Fréchet derivative in time-frequency domain, 80th EAGE conference and Exhibition 2018.

[54] Ogilvy, J.A., 1991, Theory of wave scattering form random rough surfaces, Adam Hilger.

[55] Chen, Guoxin, Ru-Shan Wu, Yuqing Wang and Shengchang Chen, 2018b, Multi-scale signed envelope inversion, Journal of applied Geophysics, 153, 113-126..

[56] Candes, E.J. and C. Fernandez-Granda, 2014, Towards a Mathematical Theory of Superresolution, Com. On Pure and appl. Math, LXVII, 0906-0956.

[57] Xu G, Wang X, Xu X and Shao L, 2014, Amplitude and Phase Analysis Based on Signed Demodulation for AM-FM Signal, Journal of Computer and Communications, 2(09), 87.

[58] Taner M T, Koehler F, Sheriff R.E, 1979, Complex seismic trace analysis, Geophysics, 44(6): 1041-1063.

[59] Chen, Guoxin, Ru-Shan Wu and Shengchang Chen, 2018d, Multi-scale direct envelope inversion: algorithm and methodology for application to salt structure inversion. Earth and Space Science,submitted.

[60] Newton, R.G., 1989, Inverse Schrödinger scattering in three dimensions, Springer-Verlag.

[61] Pike, R. and P. Sabatier, 2002, Scattering and inverse scattering in pure and applied scienc, Academic Press. 